\title{
Entanglement spectrum of geometric states
}

\author{
Wu-zhong Guo \\ School of Physics, Huazhong University of Science and Technology, \\ Luoyu Road 1037, Wuhan, Hubei 430074, China \\ E-mail: wuzhong@hust.edu.cn
}

ABSTRACT: The reduced density matrix of a given subsystem, denoted by $\rho_{A}$, contains the information on subregion duality in a holographic theory. We may extract the information by using the spectrum (eigenvalue) of the matrix, called entanglement spectrum in this paper. We evaluate the density of eigenstates, one-point and two-point correlation functions in the microcanonical ensemble state $\rho_{A, m}$ associated with an eigenvalue $\lambda$ for some examples, including a single interval and two intervals in vacuum state of $2 \mathrm{D}$ CFTs. We find there exists a microcanonical ensemble state with $\lambda_{0}$ which can be seen as an approximate state of $\rho_{A}$. The parameter $\lambda_{0}$ is obtained in the two examples. For a general geometric state, the approximate microcanonical ensemble state also exists. The parameter $\lambda_{0}$ is associated with the entanglement entropy of $A$ and Rényi entropy in the limit $n \rightarrow \infty$. As an application of the above conclusion we reform the equality case of the Araki-Lieb inequality of the entanglement entropies of two intervals in vacuum state of 2D CFTs as conditions of Holevo information. We show the constraints on the eigenstates. Finally, we point out some unsolved problems and their significance on understanding the geometric states.

KeYwords: AdS-CFT Correspondence, Conformal Field Theory

ARXIV EPRINT: 2008.12430 


\section{Contents}

1 Introduction 2

2 Entanglement spectrum and microcanonical ensemble state 4

2.1 Density of eigenstates 4

2.1.1 One-point functions 5

2.2 Example: one interval in the vacuum state of 2D CFTs 6

2.2.1 Density of eigestates 6

2.2.2 One-point functions of quasi-primary operators in the vacuum conformal family $\quad 7$

2.2.3 General operators 8

2.2.4 Modular Hamiltonian 9

$\begin{array}{ll}2.2 .5 \text { Two-point functions } & 10\end{array}$

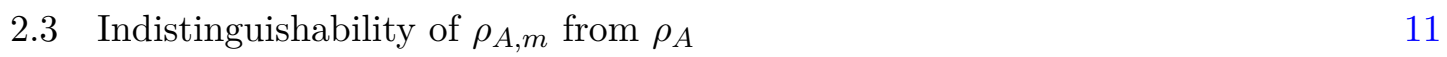

3 Entanglement spectrum of geometric states $\quad 12$

$\begin{array}{ll}3.1 \text { Two intervals in vacuum state } & 12\end{array}$

$\begin{array}{lll}3.2 & \text { Arbitrary geometric states } & 14\end{array}$

3.2.1 The gravity dual of Rényi entropy 14

$\begin{array}{ll}\text { 3.2.2 Density of eigenstates and microcanonical ensemble } & 15\end{array}$

3.2.3 Correlation functions in microcanonical ensemble state 16

$\begin{array}{lll}3.3 & \text { Higher dimensions } & 17\end{array}$

4 Applications of the microcanonical ensemble states $\quad 18$

$\begin{array}{ll}4.1 \text { Holevo information } & 18\end{array}$

$\begin{array}{ll}4.2 & \text { Operational meaning of the Holevo information } \\ \end{array}$

4.3 Holevo information $\chi\left(\rho_{A_{3}}\right) \quad 20$

4.4 Constraints on one-point functions of quasi-primary operators 21

5 Conclusions and discussions $\quad 22$

5.1 Transition between distinguishability and indistinguishability 24

5.2 A possible geometric explanation of Holevo information 24

$\begin{array}{lll}5.3 & \text { On construction of new geometric states } & 25\end{array}$

A More examples of one-point functions of quasi-primary operators $\quad 26$

$\begin{array}{ll}\text { B Higher order corrections of two-point functions } & 27\end{array}$

$\begin{array}{ll}\text { C Calculations for } d=4 & 28\end{array}$

$\begin{array}{lr}\text { D Review of short interval expansion } & 29\end{array}$ 


\section{Introduction}

For a quantum field theory with a gravity dual, there exists some states that can be effectivelly described by a classical geometry in the semi-classical limit $G \rightarrow 0$. We call these states geometric states. These states should show special properties, for example the probes, such as correlation functions, in geometric states should be well-defined in the limit $G \rightarrow 0$. Among all the probes there exists some special ones that can be associated with geometric objects in the bulk. We may call them geometric probes.

At present we still have no methods to judge whether a state is geometric or not. But we can use the geometric probes to detect the properties of geometric states. Quantum entanglement is a useful concept to understand the geometry. Among various measures of entanglement, entanglement entropy (EE) of a subsystem $A$ is the most useful one since the discovery of the Ryu-Takayanagi (RT) formula [1]

$$
S_{A}=\frac{\min \left(\operatorname{Area}\left\{\gamma_{A}\right\}\right)}{4 G},
$$

where $S_{A}$ is the EE of the subsystem $A, \gamma_{A}$ is the bulk surface that is homologous to $A$. By using this simple relation one may obtain fruitful results which help us to catch the properties of the geometric states. Motivated by the holographic EE, in $[2,3]$ the author finds the secret relation between quantum entanglement and connectivity of spacetime. Another way to understand geometric states is using the analogy between tensor networks and the geometry of spatial slice of AdS/CFT [4]. The geometric states can also be constrained by using the inequality of entropy. In [5] the authors show the so-called tripartite information (a linear combinations of EEs of arbitrary three regions) in the geometric states should be non-negative, thus give strong constraints on the geometric states. Direct construction of geometric states as coherent state are studied in [6]-[8], see also [9]-[13]. On the field theory side we find a series of necessary conditions on expectation values of quasi-primary operators in $\mathrm{AdS}_{3} / \mathrm{CFT}_{2}$ by using the scaling behavior of large $c$ limit [14].

Here we only list some attempts to characterize various aspects of geometric states. Many of them are associated with EE. But EE as a functional of the reduced density matrix $\rho_{A}$ only contains limited information of $\rho_{A}$. In this paper one of our motivation is to study the spectrum of $\rho_{A}$ for the geometric states. We will shortly call them entanglement spectrum following [15], in which it is used to identify topological order. In this paper we will also use the term "eigenvalue" instead of spectrum, though in general they may be different.

Generally, the density matrix $\rho_{A}$ has the following spectrum decomposition

$$
\rho_{A}=\sum_{i} \lambda_{i}\left|\lambda_{i}\right\rangle_{A}{ }_{A}\left\langle\lambda_{i}\right|,
$$

where $\left|\lambda_{i}\right\rangle_{A}$ are generally degenerate. The maximal eigenvlue of $\rho_{A}$ is $\lambda_{m}$. We can parameterize the eigenvalue $\lambda$ as $\lambda=\lambda_{m} e^{-t}$ with $t \in[0,+\infty)$. The distributions of entanglement spectrum can be obtained if one knows the Rényi entropy $S_{A}^{(n)}$ of all the index $n$. This is done in [16] for a single interval in the vacuum state of 2D CFTs. The results can be easily generalized to the cases that are studied in [17]. In this paper we will discuss more 
non-trivial examples. Further, by using the method of inverse Laplace transformation similar with [18-20], we also study the correlation functions in the so-called microcanonical ensemble states $\rho_{A, m}$,

$$
\rho_{A, m}:=\frac{1}{\mathcal{P}(t)} \sum_{i}\left|\lambda_{i}\right\rangle_{A}{ }_{A}\left\langle\lambda_{i}\right| \delta\left(t_{i}-t\right)
$$

where $\mathcal{P}(t)$ is the density of eigenstate with respect to $t$. Our motivation is to explore the behavior of eigenvalues and eigenstates of $\rho_{A}$ in the large $c$ limit in 2D CFTs. Our examples include a single interval and two intervals in vacuum state. The examples show one could find a microcanonical ensemble state to be an approximate state of $\rho_{A}$ in the large $c$ limit, if the probes are located in a small region of $A$ and far away from the boundary of $A$. We make the above conclusions by directly comparing the one-point and two-point functions in $\rho_{A, m}$ with the ones in $\rho_{A}$.

The results can be generalized to arbitrary geometric states by using the fact that $S_{A}^{(n)} \sim O(c)$ or $O(1 / G)$, which follows the holographic Rényi entropy proposal in [21]. The microcanonical ensemble state are associated with the EE $S\left(\rho_{A}\right)$ and $S^{\infty}=\lim _{n \rightarrow \infty} S_{A}^{(n)}$. More preciesely, the parameter $t_{0}$ of the special microcanonical ensemble state is given by

$$
t_{0}=S\left(\rho_{A}\right)-S^{\infty}
$$

Our results actually give a general feature of the entanglement spectrum of geometric states.

One of the interesting phenomenon that one can obtain from (1.1) is the phase transition of the EE of two disconnected subsystems. Without loss of generality we will focus on two intervals in the vacuum state of $2 \mathrm{D}$ CFTs. In this paper we will choose $A_{1}=[-R,-T]$ and $A_{2}=[T, R]$ with $R>T>0$ and denote $A_{3}=[-T, T]$. By using the RT formula (1.1) one can find the critical point at $T_{0}=(3-2 \sqrt{2}) R$. For $T>T_{0}$ we have $S_{A_{1} A_{2}}=S_{A_{1}}+S_{A_{2}}$ which means $A_{1}$ and $A_{2}$ lose correlations. For the case $T<T_{0}$ we have

$$
S_{A_{1} A_{2}}=S_{A_{3}}+S_{A_{1} A_{2} A_{3}} .
$$

The equality is satisfied for the Araki-Lieb inequality $S_{A B} \geq S_{A}-S_{B}$ with $A=A_{1} A_{2}$ and $B=A_{3}$.

In this paper we also study the implication of the equality condition of Araki-Lieb inequality on the spectrum of the reduced density matrices $\rho_{A_{1} A_{2} A_{3}}$ and $\rho_{A_{3}}$. This is based on reforming the above condition as Holevo information, that is

$$
\chi\left(\rho_{A_{1} A_{2}}\right)=H\left(\lambda_{i}\right)+O\left(c^{0}\right) \text { and } \quad \chi\left(\rho_{A_{3}}\right)=0+O\left(c^{0}\right),
$$

where $H\left(\lambda_{i}\right)$ is the EE of $A, \rho_{A_{1} A_{2}}=\sum_{i} \lambda_{i} \rho_{i, A_{1} A_{2}}$ and $\rho_{A_{3}}=\sum_{i} \lambda_{i} \rho_{i, A_{3}}$ with $\rho_{i, A_{1} A_{2}}:=$ $\operatorname{tr}_{A_{3}}\left|\lambda_{i}\right\rangle_{A}{ }_{A}\left\langle\lambda_{i}\right|$ and $\rho_{i, A_{3}}:=\operatorname{tr}_{A_{1} A_{2}}\left|\lambda_{i}\right\rangle_{A}{ }_{A}\left\langle\lambda_{i}\right|$. This condition gives the constraints on measurement or quasi-primary operators of the vacuum conformal family in the single eigenstate.

Finally, we discuss the possible extensions based on present paper. One interesing question is how to find the critical point of the distinguishability and indistinguishability 
of $\rho_{A, m}$ with $t=t_{0}$ from $\rho_{A}$. We also discuss the possibility of the Holevo information as a geometric probe. At last we compare the microcanonical ensemble state in this paper with the approximate state constructed by tensor networks and the fixed-area state in the quantum error-correcting code of AdS.

\section{Entanglement spectrum and microcanonical ensemble state}

\subsection{Density of eigenstates}

For a pure state $|\psi\rangle$ a Schmidt decomposition of the subsystem $A$ and its complement $\bar{A}$ is given by

$$
|\psi\rangle=\sum_{i} \sqrt{\lambda_{i}}\left|\lambda_{i}\right\rangle_{A} \otimes\left|\bar{\lambda}_{i}\right\rangle_{\bar{A}}
$$

where $\left|\lambda_{i}\right\rangle_{A(\bar{A})} \in \mathcal{H}_{A(\bar{A})}$ satisfy ${ }_{A(\bar{A})}\left\langle\lambda_{i} \mid \lambda_{j}\right\rangle_{A(\bar{A})}=\delta_{i j}$. It is obvious that $\left|\lambda_{i}\right\rangle_{A(\bar{A})}$ are the eigenstates of $\rho_{A(\bar{A})}$. For QFTs the spectrum of $\rho_{A}$ is continous, thus the sum of the decomposition should be replaced by integration. If $|\psi\rangle=|0\rangle$ and $A$ is an interval, the eigenvalues and the corresponding eigenstates should only depend on the length of the interval by translation invariance. The reduced density matrix of $A$ is $\rho_{A}=\sum_{i} \lambda_{i}\left|\lambda_{i}\right\rangle_{A}{ }_{A}\left\langle\lambda_{i}\right|$, where $\lambda_{i}$ is the eigenvalue of $\rho_{A}$. The EE of $A$ is given by the Shannon entropy $H\left(\lambda_{i}\right):=-\sum_{i} \lambda_{i} \log \lambda_{i}$.

With the Rényi entropy $S_{A}^{(n)}:=\frac{\log \operatorname{tr} \rho_{A}^{n}}{1-n}$ of all the index $n$, one could construct density of eigenstates of the reduced density matrix $\rho_{A}$. The density of the states at the eigenvalues $\lambda$ is defined as

$$
P(\lambda):=\sum_{k} \delta\left(\lambda_{k}-\lambda\right)
$$

which satisfies the normalization condition $\int_{0}^{\lambda_{m}} d \lambda \lambda P(\lambda)=1$ with $\lambda_{m}$ is the maximal eigenvalue of $\rho_{A}$. One may calculate $P(\lambda)$ of one interval in vacuum state of $2 \mathrm{D}$ CFTs by using the method in [16]. For our motivation we will use the inverse Laplace transformation method that is mentioned in the same reference. The Laplace transformation method is used in [22] to study the entanglement sepectrum of a sphere in the vacuum state. By the definition of the Rényi entropy we can find the maximal eigenvalue $\lambda_{m}$ of $\rho_{A}$ by

$$
b:=-\log \lambda_{m}=\lim _{n \rightarrow \infty} S_{A}^{(n)} .
$$

We also have

$$
\operatorname{tr} \rho_{A}^{n}=\int_{0}^{\lambda_{m}} d \lambda \lambda^{n} P(\lambda)=e^{(1-n) S_{A}^{(n)}} .
$$

Let's parameterize $\lambda$ as $\lambda=\lambda_{m} e^{-t}$, the integral becomes the form of Laplace transformation,

$$
\int_{0}^{+\infty} P\left(\lambda_{m} e^{-t}\right) e^{-(n+1) t} d t=\lambda_{m}^{-(n+1)} e^{(1-n) S_{A}^{(n)}} .
$$

By inverse Laplace transform we have the density of eigenstates with respect to $t$

$$
\mathcal{P}(t):=P\left(\lambda_{m} e^{-t}\right) \lambda_{m} e^{-t}=\mathcal{L}^{-1}\left[\lambda_{m}^{-n} e^{(1-n) S_{A}^{(n)}}\right](t):=\frac{1}{2 \pi i} \int_{\gamma-i \infty}^{\gamma+i \infty} d n e^{n t} e^{n b+(1-n) S_{A}^{(n)}} .
$$


Using this one can get the density of eigenstates $P(\lambda)$

$$
P(\lambda)=\left.\frac{\lambda_{m}}{\lambda} \mathcal{L}^{-1}\left[\lambda_{m}^{-(n+1)} e^{(1-n) S_{A}^{(n)}}\right](t)\right|_{t \rightarrow \log \frac{\lambda_{m}}{\lambda}} .
$$

\subsubsection{One-point functions}

The one-point correlation functions are useful probes to study the properties of the eigenstates of $\rho_{A}$. For an operator $\mathcal{O}$ we define the one-point functions

$$
P_{\mathcal{O}}(\lambda):=\sum_{i}{ }_{A}\left\langle\lambda_{i}|\mathcal{O}| \lambda_{i}\right\rangle_{A} \delta\left(\lambda_{i}-\lambda\right) .
$$

The density of eigenstate $P(\lambda)$ is a special case with $\mathcal{O}=I$. We assume the operator $\mathcal{O}$ is located in the region $A$. So we have

$$
\operatorname{tr}\left(\rho_{A} \mathcal{O}\right)=\sum_{j}{ }_{A}\left\langle\lambda_{j}|\mathcal{O}| \lambda_{j}\right\rangle_{A}=\int_{0}^{\lambda_{m}} d \lambda \lambda P_{\mathcal{O}}(\lambda),
$$

and

$$
\operatorname{tr}\left(\rho_{A}^{n} \mathcal{O}\right):=\int_{0}^{\lambda_{m}} d \lambda \lambda^{n} P_{\mathcal{O}}(\lambda)
$$

Taking $\lambda=\lambda_{m} e^{-t}$, the above integral becomes

$$
\operatorname{tr}\left(\rho_{A}^{n} \mathcal{O}\right)=\lambda_{m}^{n+1} \int_{0}^{+\infty} d t e^{-(n+1) t} P_{\mathcal{O}}\left(\lambda_{m} e^{-t}\right) .
$$

By an inverse Laplace transformation we get

$$
\mathcal{P}_{\mathcal{O}}(t):=P_{\mathcal{O}}\left(\lambda_{m} e^{-t}\right) \lambda_{m} e^{-t}=\mathcal{L}^{-1}\left[\lambda_{m}^{-n} \operatorname{tr}\left(\rho_{A}^{n} \mathcal{O}\right)\right](t) .
$$

The one-point functions $P_{\mathcal{O}}(\lambda)=\mathcal{P}_{\mathcal{O}}(t) /\left.\lambda\right|_{t \rightarrow \log \left(\lambda_{m} / \lambda\right)}$. To evaluate them one needs $\operatorname{tr}\left(\rho_{A}^{n} \mathcal{O}\right)$. In the path integral formalism $\operatorname{tr}\left(\rho_{A}^{n}\right)=Z_{n}(A) / Z_{1}^{n}$, where $Z_{n}(A)$ is the path integral on the n-sheeted surface $\mathcal{R}_{n}$. By the definition of the one-point function of $\mathcal{O}$ on the surface $\mathcal{R}_{n}$ we have

$$
\langle\mathcal{O}\rangle_{\mathcal{R}_{n}}:=\frac{\int_{\mathcal{R}_{n}} D \phi \mathcal{O} e^{-I_{E}}}{\int_{\mathcal{R}_{n}} D \phi e^{-I_{E}}}=\frac{\operatorname{tr}\left(\rho_{A}^{n} \mathcal{O}\right)}{\operatorname{tr} \rho_{A}^{n}}
$$

Thus we have

$$
\begin{aligned}
\mathcal{P}_{\mathcal{O}}(t) & =\mathcal{L}^{-1}\left[\lambda_{m}^{-n} e^{(1-n) S_{A}^{(n)}}\langle\mathcal{O}\rangle_{\mathcal{R}_{n}}\right](t) \\
& =\frac{1}{2 \pi i} \int_{\gamma-i \infty}^{\gamma+i \infty} d n e^{n t} e^{n b+(1-n) S_{A}^{(n)}}\langle\mathcal{O}\rangle_{\mathcal{R}_{n}} .
\end{aligned}
$$

For a single interval in some special states of 2D CFTs one may calculate $\langle\mathcal{O}\rangle_{\mathcal{R}_{n}}$. We will show some examples later. In the limit $n \rightarrow \infty$ the domain contribution to $\operatorname{tr}\left(\rho_{A}^{n} \mathcal{O}\right)$ is given by

$$
\lambda_{m}^{n} \sum_{m_{i}}{ }_{A}\left\langle\lambda_{m_{i}}|\mathcal{O}| \lambda_{m_{i}}\right\rangle_{A} \delta\left(\lambda_{m_{i}}-\lambda_{m}\right) .
$$


By using (2.13) we find

$$
\sum_{m_{i}}{ }_{A}\left\langle\lambda_{m_{i}}|\mathcal{O}| \lambda_{m_{i}}\right\rangle_{A} \delta\left(\lambda_{m_{i}}-\lambda_{m}\right)=\lim _{n \rightarrow \infty}\langle\mathcal{O}\rangle_{\mathcal{R}_{n}}
$$

To calculate the one-point functions in other eigenstates one should evaluate the inverse Laplace transformation. We define the average one-point functions

$$
\overline{\mathcal{P}}_{\mathcal{O}}(t):=\frac{\mathcal{P}_{\mathcal{O}}(t)}{\mathcal{P}(t)} .
$$

\subsection{Example: one interval in the vacuum state of 2D CFTs}

\subsubsection{Density of eigestates}

Once Rényi entropy of all the index $n$ are known we could obtain the density of eigenstates by using (2.6). For 2D CFTs there are several known examples including a single interval with length $l$ in the vacuum state or finite temperature and half infinite line in the regularized boundary states [17]. For all these examples the modular Hamiltonian $H_{A}$ can be written as local integral over energy density in the subsystem $A$,

$$
H_{A}=\int_{A} d x f(x) T_{00}(x)
$$

where $f(x)$ depends on the situation we are considering. For all the examples the Rényi entropy is like the form

$$
S_{A}^{(n)}=\left(1+\frac{1}{n}\right) b
$$

Let's consider some examples. $\quad b=\frac{c}{6} \log \frac{l}{\epsilon}$ if the size of system is infinite, where $\epsilon$ is the UV cut-off. $b=\frac{c}{12} \log \frac{2 l}{\epsilon}$ if $A$ is the interval at the end of a semi-infinite line. $b=$ $\frac{c}{6} \log \left[\frac{\beta}{\pi \epsilon} \sinh \left(\frac{\pi l}{\beta}\right)\right]$ if the system is in the canonical ensemble thermal state with inverse temperature $\beta$.

By using (2.6) and (2.19) we have

$$
\mathcal{P}(t)=\delta(t)+\frac{\sqrt{b} I_{1}(2 \sqrt{b t})}{\sqrt{t}} H(t)
$$

where $H(t)$ is the Heaviside step function, $I_{n}(x)$ is the modified Bessel function of the first kind. One can check it satisfies the normalization condition

$$
\operatorname{tr} \rho_{A}=\int_{0}^{+\infty} \mathcal{P}(t) \lambda_{m} e^{-t} d t=1
$$

and the EE

$$
S_{A}=-\int_{0}^{+\infty} d t\left(\lambda_{m} e^{-t}\right) \log \left(\lambda_{m} e^{-t}\right) \mathcal{P}(t)=2 b .
$$

In this paper we are interested in the behavior of $\mathcal{P}(t)$ in the large $c$ limit. By using the fact $I_{n}(x) \simeq \frac{e^{x}}{\sqrt{2 \pi x}}$ in the large $x$ limit, $\mathcal{P}(t)$ can be approximated by

$$
\mathcal{P}(t) \simeq \delta(t)+\frac{b e^{2 \sqrt{b t}}}{\sqrt{4 \pi}(b t)^{3 / 4}} H(t) .
$$


For $t=b$ or equally $\lambda=\lambda_{0}:=e^{-2 b}$ we find $\mathcal{P}(t) \sim e^{2 b}$. The entropy of the state $\rho_{A, m}$ is $S_{A, m} \simeq 2 b+o(c)$ Here we define the microcanonical ensemble state

$$
\rho_{A, m}=\frac{1}{\mathcal{P}(t)} \sum_{i}\left|\lambda_{i}\right\rangle_{A}{ }_{A}\left\langle\lambda_{i}\right| \delta\left(t_{i}-t\right),
$$

with $t_{i}=-\log \left(\lambda_{i} / \lambda_{m}\right)$. The above results show the EE of the microcanonical ensemble state with $t=b$ is equal to the EE of $A$ at the leading order of $c$. This motivates us to propose the microcanonical ensemble state $\rho_{A, m}$ with $t=b$ can be an approximate state of $\rho_{A}$.

\subsubsection{One-point functions of quasi-primary operators in the vacuum conformal family}

The quasi-primary operators in the vacuum conformal family include the stress energy tensor $T(w)$ with conformal dimension $h_{T}=2, \mathcal{A}:=(T T)-\frac{3}{10} \partial^{2} T$ with conformal dimension $h_{\mathcal{A}}=4$ and so on. In this section for simplicity we only consider $T$ and $\mathcal{A}$. One could refer to $[23,24]$ for more details on these operators.

By the conformal transformation $z=f(w)=\left(\frac{w+R}{w-R}\right)^{1 / n}$ the n-sheet surface $\mathcal{R}_{n}$ is mapped to complex $z$-plane. One may get one-point function of $T$ and $\mathcal{A}$ in $\mathcal{R}_{n}$

$$
\langle T\rangle_{\mathcal{R}_{n}}=\frac{c}{6} \frac{n^{2}-1}{n^{2}} \frac{R^{2}}{\left(R^{2}-w^{2}\right)^{2}}, \quad \text { and } \quad\langle\mathcal{A}\rangle_{\mathcal{R}_{n}}=\frac{c(5 c+22)}{180} \frac{\left(n^{2}-1\right)^{2}}{n^{4}} \frac{R^{4}}{\left(R^{2}-w^{2}\right)^{4}}
$$

Taking $\langle T\rangle_{\mathcal{R}_{n}}$ into (2.14) we get

$$
\mathcal{P}_{T}(t)=\frac{c R^{2}}{6\left(R^{2}-w^{2}\right)^{2}} \delta(t)+\frac{c R^{2}}{6\left(R^{2}-w^{2}\right)^{2}} \frac{\sqrt{b} I_{1}(2 \sqrt{b t})}{\sqrt{t}}\left(1-\frac{t}{b}\right) H(t) .
$$

The first term gives the contribution from the maximal eigenvalue $\lambda_{m}$, which is consistent with (2.16). For $t \neq 0$ or equally $\lambda \neq \lambda_{m}$ the average one-point functions of $T$ is

$$
\overline{\mathcal{P}}_{T}(t)=\frac{c R^{2}}{6\left(R^{2}-w^{2}\right)^{2}}\left(1-\frac{t}{b}\right) .
$$

Similarly, we can derive

$$
\mathcal{P}_{\mathcal{A}}(t)=\frac{c(5 c+22) R^{4}}{180\left(R^{2}-w^{2}\right)^{4}} \delta(t)+\frac{c(5 c+22) R^{4}}{180\left(R^{2}-w^{2}\right)^{4}} \frac{\sqrt{b} I_{1}(2 \sqrt{b t})}{\sqrt{t}}\left[1-2 \frac{t}{b}+\frac{t^{2}}{b^{2}} \frac{I_{3}(2 \sqrt{b t})}{I_{1}(2 \sqrt{b t})}\right] H(t) .
$$

For $t \neq 0$ the average one-point function is

$$
\overline{\mathcal{P}}_{\mathcal{A}}(t)=\frac{c(5 c+22) R^{4}}{180\left(R^{2}-w^{2}\right)^{4}}\left[1-2 \frac{t}{b}+\frac{t^{2}}{b^{2}} \frac{I_{3}(2 \sqrt{b t})}{I_{1}(2 \sqrt{b t})}\right] .
$$

Note that in the limit $c \rightarrow \infty$ the above expression can be approximated by

$$
\overline{\mathcal{P}}_{\mathcal{A}}(t) \simeq \frac{c(5 c+22) R^{4}}{180\left(R^{2}-w^{2}\right)^{4}}\left(1-\frac{t}{b}\right)^{2}
$$

by using the fact $I_{3}(2 \sqrt{b t}) / I_{1}(2 \sqrt{b t}) \rightarrow 1$. 
Both of the two examples show the average one-point function $\overline{\mathcal{P}}(t)$ is divergent at $R$ and $-R$, which are the boundary of the subsystem $A$. Actually this should be a general result for any operators. To obtain a well defined eigenstates and eigenvalues of $H_{A}$ we should make some a UV cut-off $\epsilon$ near the ending point of subsystem $A$ [17]. The divergence of one-point functions is closely related to the regularization. In the large $c$ limit the onepoint functions are vanishing in the eigenstates $t_{0}=b$ or $\lambda_{0}=\lambda_{m}^{2}$. This is consistent with our expectation that the microcanonical ensemble state $\rho_{A, m}(2.24)$ can approximate $\rho_{A}$ since $\operatorname{tr}\left(\rho_{A} T\right), \operatorname{tr}\left(\rho_{A} \mathcal{A}\right)=0$.

\subsubsection{General operators}

In appendix $\mathrm{A}$ we calculate more examples of the one-point functions of quasi-parimary operator in vacuum conformal family. All these examples show $\mathcal{P}_{\mathcal{O}}(t)$ is vanishing for $t=b$ in the large $c$ limit. In this section we would like to show the conclusion is true for any quasi-primary operators in vacuum family.

Under the conformal transformation $z=f(w)$ a general quasi-parimary operator $\mathcal{X}(w)$ with conformal dimension $h_{\mathcal{X}}$ would transform as the following,

$$
\mathcal{X}(w)=f^{\prime}(w)^{h_{\mathcal{X}}} \mathcal{X}(z)+\sum_{\mathcal{Y}, p} F_{\mathcal{Y}, p}[f(w)] \partial^{p} \mathcal{Y}[f(w)]+S[f(w)]
$$

where the sum over $\mathcal{Y}$ includes all the quasi-primary operators with conformal dimension $h_{\mathcal{Y}} \leq h_{\mathcal{X}}, F_{\mathcal{Y}, p}[f(w)]$ and $S[f(w)]$ are functionals of $f(w)$ and its derivatives. By the conformal map $f(w)=\left(\frac{w+R}{w-R}\right)^{1 / n}$ the image of n-sheeted surface $\mathcal{R}_{n}$ is complex $z$-plane $\mathcal{C}$. By symmetry we have $\langle\mathcal{Y}(z)\rangle_{\mathcal{C}}=0$. This means $\langle\mathcal{X}(w)\rangle_{\mathcal{R}_{n}}=S[f(w)]$. Moreover, $S[f(w)]$ are composed by the Schwarzian derivative $s(w)$ and its derivatives, $s^{\prime}(w), s^{\prime \prime}(w)$ and so on. By the definition of $s(w)$ we have

$$
s(w)=\frac{\left(n^{2}-1\right)}{n^{2}} \frac{2 R^{2}}{\left(R^{2}-w^{2}\right)^{2}} .
$$

By induction one can expand $S[f(w)]$ as

$$
S[f(w)]=\sum_{I=1}^{h_{\mathcal{X}} / 2} S_{I}\left(\frac{n^{2}-1}{n^{2}}\right)^{I},
$$

where $S_{I}$ don't depend on $n$. The lower bound of the sum is not 0 since we expect $S[f(w)]$ is vanishing in the limit $n \rightarrow 1$. By using (2.12) we can derive the one-point functions of $\mathcal{X}$,

$$
\mathcal{P}_{\mathcal{X}}(t)=\frac{1}{2 \pi i} \int_{\gamma-i \infty}^{\gamma+i \infty} d n e^{n t+\frac{b}{n}} S[f(w)]
$$

Since the inverse Laplace transformation is linear, to obtain the above result we only need to evaluate the $I$-th term

$$
\mathcal{P}_{\mathcal{X}, I}(t):=\frac{1}{2 \pi i} \int_{\gamma-i \infty}^{\gamma+i \infty} d n e^{n t+\frac{b}{n}}\left(\frac{n^{2}-1}{n^{2}}\right)^{I}=\frac{1}{2 \pi i} \int_{\gamma-i \infty}^{\gamma+i \infty} d n e^{n t+\frac{b}{n}+I \log \left(\frac{n^{2}-1}{n^{2}}\right)} .
$$


For a given $I$ one could analytically calculate the integration by making derivatives on $\mathcal{P}(t)$ with respect to $b$. However, it is not easy to find a formula for any $I$. We assume $t \sim c$ which is very large. The above integral is dominated by a saddle point with

$$
n \simeq \sqrt{\frac{b}{t}}+o\left(c^{0}\right)
$$

So the saddle point approximation of the integral is

$$
\mathcal{P}_{\mathcal{X}, I}(t) \simeq \sqrt{\frac{b}{t}} e^{2 \sqrt{b t}}\left(1-\frac{t}{b}\right)^{I} .
$$

We get the one-point function

$$
\mathcal{P}_{\mathcal{X}}(t)=\sum_{I=1}^{h_{\mathcal{X}} / 2} S_{I} \mathcal{P}_{\mathcal{X}, I}(t) .
$$

For $t=b$ we conclude that the one-point functions $\mathcal{P}_{\mathcal{X}}(t)$ is vanishing for any quasi-primary operators in the vacuum conformal family.

\subsubsection{Modular Hamiltonian}

For the examples we consider the modular Hamiltonian can be written as a local integral over energy density in the subsystem $A$ (2.18). One could get the function $f(x)$ by the method used in [17]. Let's consider $A$ to be a single interval on infinite line. The modular Hamiltonian is given by

$$
H_{A}=-\int_{-R}^{R} d w \frac{R^{2}-w^{2}}{2 R} T(w)-\int_{-R}^{R} d \bar{w} \frac{R^{2}-\bar{w}^{2}}{2 R} \bar{T}(\bar{w}) .
$$

We would like to calculate

$$
\mathcal{P}_{H_{A}}(t):=\sum_{i}{ }_{A}\left\langle\lambda_{i}\left|H_{A}\right| \lambda_{i}\right\rangle_{A} \delta\left(t_{i}-t\right)
$$

by using (2.27). Taking (2.27) and (2.39) into the above equation, we find the integral is divergent near the boundary point of $A$. Therefore, we should make some regularization.

To avoid sharp bipartition of Hilbert space $\mathcal{H}$ into $\mathcal{H}_{A}$ and $\mathcal{H}_{\bar{A}}$, one should put a UV cut-off around the boundary of $A$ and $\bar{A}$. In the Euclidean path integral expression this is done by introducing a slice around the boundary point of $A$ and $\bar{A}$. The effect on the modular Hamiltonian is changing the integral region to be $[-R+\epsilon, R-\epsilon]$.

For $t \neq 0$ by using (2.27) and (2.39) we have

$$
\mathcal{P}_{H_{A}}(t)=-\int_{-R+\epsilon}^{R-\epsilon} d w \frac{R^{2}-w^{2}}{2 R} \mathcal{P}_{T}(t)-\int_{-R+\epsilon}^{R-\epsilon} d \bar{w} \frac{R^{2}-\bar{w}^{2}}{2 R} \mathcal{P}_{\bar{T}}(t)=(t-b) \mathcal{P}(t),
$$

where we have used $b=\frac{c}{6} \log \frac{2 R}{\epsilon}$. For $t=0$ by using (2.16) we have

$$
\mathcal{P}_{H_{A}}(t=0)=b .
$$


With this we can calculate $\operatorname{tre}^{-H_{A}}$ by

$$
\operatorname{tr}\left(e^{-H_{A}}\right)=e^{-b}+\int_{0}^{+\infty} d t e^{b-t} \mathcal{P}(t)=e^{2 b},
$$

where the first term comes from the contribution of $t=0$. Therefore, the normalized reduced density matrix $\rho_{A}$ is

$$
\rho_{A}=e^{-H_{A}-2 b}
$$

Notice that the spectrum of $H_{A}+2 b$ is $t+b$, which is positive. So the operator $H_{A}+2 b$ is a positive operator.

One may construct other operators like the modular Hamiltonian, such as

$$
Q_{\mathcal{A}}=\int_{-R}^{R} d w \frac{\left(R^{2}-w^{2}\right)^{3}}{R^{3}} \mathcal{A}(w)+\int_{-R}^{R} d \bar{w} \frac{\left(R^{2}-\bar{w}^{2}\right)^{3}}{R^{3}} \overline{\mathcal{A}}(\bar{w}) .
$$

The expectation value of this operator in the microcanonical ensemble state $(2.24)$ is

$$
\mathcal{P}_{Q_{\mathcal{A}}}(t) \simeq \frac{b c}{3}\left(1-\frac{t}{b}\right)^{2} \mathcal{P}(t)
$$

where we have assumed the large $c$ limit and used (2.30). It seems the operators $Q_{A}$ and $H_{A}$ don't have common eigenstates $\left|\lambda_{i}\right\rangle_{A}$. However, $Q_{A}$ still show very similar behavior as the modular Hamiltonian. One also could construct other similar operators by $\mathcal{B}, \mathcal{D}$, etc. It is interesting to explore these kinds of operators in the future.

\subsubsection{Two-point functions}

The one-point functions of primary operators are vanishing for a single interval in infinite line because $\langle O\rangle_{\mathcal{R}_{n}}=0$. For the interval at the end of a semi-infinite line or in the regularized boundary state the one-point functions are non-vanishing since the one point function in these two states can be associated with two-point functions on the complex plane. In this section we would like to discuss the two-point functions as probes to detect the microcanonical state $\rho_{A, m}$.

The two-point functions are defined as

$$
\mathcal{P}_{\mathcal{O O}}(t):=\sum_{i}{ }_{A}\left\langle\lambda_{i}|\mathcal{O}(x) \mathcal{O}(y)| \lambda_{i}\right\rangle_{A} \delta\left(t_{i}-t\right) .
$$

The average two-point function is $\overline{\mathcal{P}}_{\mathcal{O O}}(t):=\mathcal{P}_{\mathcal{O O}}(t) / \mathcal{P}(t)$. Without loss of generality we choose $0<x<R$ and $y=-x$. To calculate the two-point functions we need

$$
\begin{aligned}
\langle\mathcal{O}(x) \mathcal{O}(-x)\rangle_{\mathcal{R}_{n}} & =f^{\prime}(x)^{h_{\mathcal{O}}} f^{\prime}(-x)^{h_{\mathcal{O}}}(f(x)-f(-x))^{-2 h_{\mathcal{O}}} \\
& =\left(\frac{2 R}{n\left(R^{2}-x^{2}\right)}\right)^{2 h_{\mathcal{O}}}\left(\xi^{-\frac{1}{2 n}}-\xi^{\frac{1}{2 n}}\right)^{-2 h_{\mathcal{O}}},
\end{aligned}
$$

where $\xi:=\left(\frac{R-x}{R+x}\right)^{2}$. By using (2.12) and (2.13) and the above result we can obtain

$$
\mathcal{P}_{\mathcal{O O}}(t)=\frac{1}{2 \pi i} \int_{\gamma-i \infty}^{\gamma+i \infty} d n e^{n t+\frac{b}{n}}\langle\mathcal{O}(x) \mathcal{O}(-x)\rangle_{\mathcal{R}_{n}}
$$


We cannot find an analytical result of the inverse Laplace transformation. If the distance between the two operators is small, i.e., $x \ll R$, we have $\xi \sim 1$ and

$$
\langle\mathcal{O}(x) \mathcal{O}(-x)\rangle_{\mathcal{R}_{n}}=(2 x)^{-2 h_{\mathcal{O}}}+\frac{h_{\mathcal{O}}\left(n^{2}-1\right)(2 x)^{2-2 h_{\mathcal{O}}}}{3 n^{2} R^{2}}+o\left(x^{2-2 h_{\mathcal{O}}}\right) .
$$

For $t \neq 0$ this gives

$$
\mathcal{P}_{\mathcal{O O}}(t)=\mathcal{P}(t)(2 x)^{-2 h_{\mathcal{O}}}\left(1+\frac{h_{\mathcal{O}}(2 x)^{2}}{3 R^{2}}\left(1-\frac{t}{b}\right)+o\left(x^{2}\right)\right) .
$$

Here we only list the results upto $O\left(x^{2}\right)$. In appendix B we calculate the two-point functions upto $O\left(x^{6}\right)$. An important feature of the results upto $O\left(x^{6}\right)$ is

$$
\overline{\mathcal{P}}_{\mathcal{O O}}(t=b)=(2 x)^{-2 h_{\mathcal{O}}}+o\left(x^{6}\right),
$$

in the large $c$ limit, which is consistent with the two point correlation function in vacuum state $\langle O(x) O(-x)\rangle=(2 x)^{-2 h_{\mathcal{O}}}$ upto $O\left(x^{6}\right)$. This further supports our expectation that the mircocanonical state $\rho_{A, m}$ can approximate the reduced density state $\rho_{A}$ in the large $c$ limit.

If the distance between two operators is large, i.e., $x \sim R$, we have $\xi \sim 0$ and

$$
\langle\mathcal{O}(x) \mathcal{O}(-x)\rangle_{\mathcal{R}_{n}} \simeq\left(\frac{2 R}{n\left(R^{2}-x^{2}\right)}\right)^{2 h_{\mathcal{O}}} \xi^{\frac{h_{\mathcal{O}}}{n}}
$$

For $t \neq 0$ we get

$$
\left.\mathcal{P}_{\mathcal{O O}}(t)=\left(\frac{2 R}{R^{2}-x^{2}}\right)^{2 h_{\mathcal{O}}}\left(\frac{b+h_{\mathcal{O}} \log \frac{R-x}{R+x}}{t}\right)^{\frac{1-2 h_{\mathcal{O}}}{2}} I_{2 h-1}\left(2 \sqrt{t\left(b+h_{\mathcal{O}} \log \frac{R-x}{R+x}\right.}\right)\right) .
$$

Even if taking $t=b$ we find the two-point functions are not same as $\langle\mathcal{O}(x) \mathcal{O}(-x)\rangle$.

\subsection{Indistinguishability of $\rho_{A, m}$ from $\rho_{A}$}

In the previous sections we calculate the one-point functions and two-point functions in the microcanonical state $\rho_{A, m}$. If taking the large $c$ limit, we find the one-point functions in the state $\rho_{A, m}$ with $t=b$ is vanishing. The two-point correlation functions of primary operators are equal to the ones in $\rho_{A}$ upto $O\left(x^{6}\right)$, where $2 x$ is the distance of the two operators. These are consistent with our expectation that $\rho_{A, m}$ with $t=b$ can be seen as an approximate state of $\rho_{A}$ in the large $c$ limit. However, if the distance between the two operators are large $x \sim R$, the two-point functions $\mathcal{P}_{\mathcal{O O}}(t)$ are different from the ones in $\rho_{A}$. Therefore, a reasonable explanation of these results is $\rho_{A, m}$ is indistinguishable from $\rho_{A}$ in large $c$ limit only if the probes are located in a small region comparing with $R$, that is $T / R \ll 1$. One could define a reduced density matrix $\rho_{A_{3}, m}:=\operatorname{tr}_{A_{1} A_{2}} \rho_{A, m}$. In the case $T / R \ll 1$ one could show that the distance between $\rho_{A_{3}, m}$ and $\rho_{A_{3}}$ would approach to 0 in the large $c$ limit, i.e.,

$$
\lim _{c \rightarrow \infty} d\left(\rho_{A_{3}, m}, \rho_{A_{3}}\right) \rightarrow 0,
$$

where $d(\rho, \sigma)$ are the quantities that characterize the distance between two density matrices, such as trace distance, relative entropy, etc. There are some studies on relative 
entropy and trace distance in CFTs, see [25]-[32]. Here we will not dicuss the details of the distance, but one could calculate the trace distance and relative entropy by using our results in previous sections.

However, in the case $T / R \sim 1$ the two states $\rho_{A, m}$ and $\rho_{A}$ are distinguishable, for example one could use the two-point function with distance $2 x$ as a probe. This phenomenon also appears in the discussion of the canonical ensemble thermal state and the microcanonical state. One could show the reduced density matrices of a small subsystem are indistinguishable for the two ensemble states ( see the supplemental material of [33]). However, for large subsystem there exists many probes to distinguish them. One of them is the Rényi entropy $[34,35]$. Here we can also use the Rényi entropy to distinguish $\rho_{A, m}$ from $\rho_{A}$. Specially, for the system $A$ their Rényi entropies are different,

$$
S^{(n)}\left(\rho_{A, m}\right)=2 b, \quad S^{(n)}\left(\rho_{A}\right)=\left(1+\frac{1}{n}\right) b .
$$

In a summary, $\rho_{A_{3}, m}$ and $\rho_{A_{3}}$ are indistinguishable if the size of $A_{3}$ is small enough, but they are distinguishable if the size of $A_{3}$ is large.

\section{Entanglement spectrum of geometric states}

In this section we will generalize the results of the single interval example to arbitrary geometric states.

\subsection{Two intervals in vacuum state}

Before we discuss the general case, let's see a non-trivial example. Without loss of generality we could consider the two intervals $A_{1}$ and $A_{2}$ as mentioned in the introduction. Let's define the Rényi mutual information

$$
I^{(n)}\left(\rho_{A_{1} A_{2}}\right):=S^{(n)}\left(\rho_{A_{1}}\right)+S^{(n)}\left(\rho_{A_{2}}\right)-S^{(n)}\left(\rho_{A_{1} A_{2}}\right) .
$$

Take $n \rightarrow 1$ we get the mutual information $I\left(\rho_{A_{1} A_{2}}\right)$. By conformal symmetry one can show the Rényi mutual information $I^{(n)}\left(\rho_{A_{1} A_{2}}\right)$ only depends on the cross ratio $\eta:=\left(\frac{1-T / R}{1+T / R}\right)^{2}$. Let's denote $I^{(n)}\left(\rho_{A_{1} A_{2}}\right)$ to be $I_{n}(\eta)$. For $T>(3-2 \sqrt{2}) R$ we have $I\left(\rho_{A_{1} A_{2}}\right)=O\left(c^{0}\right)$, which means

$$
\rho_{A_{1} A_{2}}=\rho_{A_{1}} \otimes \rho_{A_{2}}+\delta \rho,
$$

where $\delta \rho$ is taken as a small perturbation in the large $c$ limit. To derive the density of eigenstates of $\rho_{A_{1} A_{2}}$ one needs the Rényi entropy for any index $n$ as we have done for the single interval case. Unfortunately, we have no exact results of the Rényi entropy. But if the distance between the two interval $A_{1}$ and $A_{2}$ is large, one could perturbatively calculate the Rényi entropy by short interval method [36]-[38]. For $\eta \ll 1$ we have $S^{(n)}\left(\rho_{A_{1} A_{2}}\right)=$ $2\left(1+\frac{1}{n}\right) b-I_{n}(\eta)$, where $b:=\frac{c}{6} \log (R-T) / \epsilon$ and

$$
\begin{aligned}
I_{n}(\eta)= & \frac{c(n-1)(n+1)^{2} \eta^{2}}{144 n^{3}}+\frac{c(n-1)(n+1)^{2} \eta^{3}}{144 n^{3}}+\frac{c(n-1)(n+1)^{2}\left(1309 n^{4}-2 n^{2}-11\right) \eta^{4}}{207360 n^{7}} \\
& +o\left(\eta^{4}, c\right),
\end{aligned}
$$


where we only keep the order of $O(c)$ results upto $\eta^{4}$. One could refer to [23] for the higher order terms. Note that the above results only contain the contributions from the quasi-primary operators in the vacuum conformal family. In the theory with holographic dual we expect these operators gives the main contributions to the Rényi entropy.

We can formally write $\rho_{A_{1} A_{2}}$ as

$$
\rho_{A_{1} A_{2}}=\sum_{J} \sqrt{\Lambda_{J}}|J\rangle_{A_{1} A_{2}} A_{1} A_{2}\langle J|,
$$

and define the density of eigenstates

$$
P(\Lambda)=\sum_{J} \delta\left(\Lambda_{J}-\Lambda\right)
$$

Using the expression (3.3) one can find the maximal eigenvlue $\Lambda_{m}$ by

$$
B=-\log \Lambda_{m}=\lim _{n \rightarrow \infty} S^{(n)}\left(\rho_{A_{1} A_{2}}\right)=2 b+\delta b+o\left(\eta^{4}, c\right),
$$

where $\delta b=-\frac{c \eta^{2}\left(1309 \eta^{2}+1440 \eta+1440\right)}{207360}$. By using (2.6) we obtain

$$
\mathcal{P}(t):=P\left(\Lambda_{m} e^{-t}\right) \Lambda_{m} e^{-t}=\frac{1}{2 \pi i} \int_{\gamma-i \infty}^{\gamma+i \infty} d n e^{n t+n B+(1-n) S^{(n)}\left(\rho_{A_{1} A_{2}}\right)}
$$

With some calculations the integral becomes

$$
\mathcal{P}(t)=\frac{1}{2 \pi i} \int_{\gamma-i \infty}^{\gamma+i \infty} d n e^{n t+\frac{2 b}{n}+n \delta b-(1-n) I_{n}(\eta)} .
$$

One could evaluate this integral by the saddle point approximation. The approximation is safe if we assume $t \sim c$. We need to solve the equation

$$
t-\frac{2 b}{n^{2}}+\delta b-\frac{\partial}{\partial n}\left[(1-n) I_{n}(\eta)\right]=0 .
$$

Since we take $\eta \ll 1$ the equation can be solved perturbatively. The solution is

$$
\begin{aligned}
n_{0}= & \sqrt{\frac{2 b}{t}}-\frac{c \eta^{2}(4 b-3 t)}{576 \sqrt{2 b^{3} t}}-\frac{c \eta^{3}(4 b-3 t)}{576 \sqrt{2 b^{3} t}}-\frac{c \eta^{4}}{6635520 \sqrt{2 b^{7} t}} \\
& \times\left[80 b^{2}(524 b+c)+t^{2}(225 c-400 b)-72 t(b(434 b+5 c))+154 t^{3}\right]+o\left(\eta^{4}\right) .
\end{aligned}
$$

Taking the above equation back into (3.8), we have

$$
\mathcal{P}(t) \simeq n_{0} e^{s(t)}
$$

with

$$
\begin{aligned}
s(t)= & 2 \sqrt{2 b t}-\frac{c \eta^{2}(4 b-t) \sqrt{b t}}{288 \sqrt{2} b^{2}}-\frac{c \eta^{3}(4 b-t) \sqrt{b t}}{288 \sqrt{2} b^{2}}+\frac{c \eta^{4} \sqrt{t}}{3317760 \sqrt{2} b^{7 / 2}} \\
& \times\left[80 b^{2}(524 b+c)+t^{2}(45 c-80 b)-24 t(b(434 b+5 c))+22 t^{3}\right]+o\left(\eta^{4}\right) .
\end{aligned}
$$


Motivated by the discussions in section 2.1 we would like to find a microcanonical ensemble states similar with $\rho_{A, m}(2.24)$ with $t=t_{0}$ that can be taken as an approximate state of $\rho_{A_{1} A_{2}}$. A necessary condition for this is the entropy of the microcanonical state $\log \mathcal{P}\left(t_{0}\right)$ is consistent with the $\mathrm{EE}$ of $\rho_{A_{1} A_{2}}$. We find the non-trivial solution is

$$
t_{0}=2 b-\delta b+o\left(\eta^{4}\right) .
$$

Thus we have $s\left(t_{0}\right)=4 b+o\left(\eta^{4}\right)$ or

$$
\mathcal{P}\left(t_{0}\right) \simeq n_{0} e^{4 b+o\left(\eta^{4}\right)} .
$$

For $T<(3-2 \sqrt{2}) R$ we have $\eta \geq \frac{1}{2}$. Specially, for $T \ll(3-2 \sqrt{2}) R$ the perturbation results may broke down. However, we can use the following results [36],

$$
I_{n}(\eta)=I_{n}(1-\eta)+\frac{(n+1) c}{6 n} \log \frac{\eta}{1-\eta} .
$$

This gives

$$
S^{(n)}\left(\rho_{A_{1} A_{2}}\right)=S^{(n)}\left(\rho_{A_{1}}\right)+S^{(n)}\left(\rho_{A_{2}}\right)-I_{n}(1-\eta)-\frac{(n+1) c}{6 n} \log \frac{\eta}{1-\eta} .
$$

Note that $S^{(n)}\left(\rho_{A_{1}}\right)=S^{(n)}\left(\rho_{A_{2}}\right)=\frac{c(n+1)}{6 n} \log \frac{R-T}{\epsilon}$. The Rényi entropy can be written as

$$
S^{(n)}\left(\rho_{A_{1} A_{2}}\right)=\left(1+\frac{1}{n}\right) b_{1}+\left(1+\frac{1}{n}\right) b_{2}-I_{n}(1-\eta),
$$

with $b_{1}=\frac{c}{6} \log \frac{2 T}{\epsilon}$ and $b_{2}=\frac{c}{6} \log \frac{2 R}{\epsilon}$. The calculations are similar as we have done for the case $T>(3-2 \sqrt{2}) R$ by replacing $2 b$ with $b_{1}+b_{2}$ and $\eta$ with $1-\eta$. Therefore, $t_{0}$ is given by

$$
t_{0}=b_{1}+b_{2}-\delta b^{\prime}
$$

with $\delta b^{\prime}=-\frac{c(1-\eta)^{2}\left(1309(1-\eta)^{2}+1440(1-\eta)+1440\right)}{207360}$.

\subsection{Arbitrary geometric states}

Our previous discussions on entanglement spectrum on single interval or two intervals in vacuum states show interesting properties of the holographic theories. In this section we will generalize the discussions to arbitrary geometric states. To do that we need to know the scale behavior of Rényi entropy in the large $c$ limit.

\subsubsection{The gravity dual of Rényi entropy}

Let's first review the proposal of holographic Rényi entropy in [21]. The holographic Rényi entropy also follow an area law like the RT formula. For a subsystem $A$ it is given by

$$
n^{2} \partial_{n}\left(\frac{n-1}{n} S^{(n)}\left(\rho_{A}\right)\right)=\frac{\operatorname{Area}\left(\mathcal{B}_{n}\right)}{4 G},
$$

where $\mathcal{B}_{n}$ denotes a bulk codimension- 2 cosmic brane homologous to the boundary region $A$. The tension $T_{n}$ of $\mathcal{B}_{n}$ is associated with the Rényi index $n$ by

$$
T_{n}=\frac{n-1}{4 n G} .
$$


One may obtain the bulk geometry as well as the area of cosmic brane $\mathcal{B}_{n}$ by solving the Einstein equation with the Euclidean action $I=I_{\text {bulk }}+I_{\text {brane }}$, where $I_{\text {bulk }}$ includes the Einstein-Hilbert action and the matter field, $I_{\text {brane }}=T_{n} \int d^{y-1} \sqrt{g}$. In this paper we will not pursue the solutions for special cases. What we need is that the metrics for any geometric states should be of order $O\left(G^{0}\right)$, since the action of the cosmic brane is of order $O\left(G^{-1}\right)$ same as the bulk actions. Therefore, the area of $\mathcal{B}_{n}$ should be of order $O\left(G^{0}\right)$, which means Rényi entropy is of $O\left(G^{-1}\right)$ or $O(c)$ by $c \sim 1 / G$. The RT formula is a special case of the holographic Rényi entropy formula in the probe limit $n \rightarrow 1$.

In the limit $n \rightarrow \infty$ the tension $T_{n} \rightarrow T_{\infty}:=\frac{1}{4 G}$. By considering the back reaction of the cosmic brane with the tension $T_{\infty}$ one could obain the holographic Rényi entropy $S^{\infty}$. Near the proble limit $n \rightarrow 1$ one could perturbatively calculate the holographic Rényi entropy. We expect the following expansion,

$$
S^{(n)}\left(\rho_{A}\right)=S\left(\rho_{A}\right)+\sum_{k=1}(1-n)^{k} b_{k}
$$

where $S\left(\rho_{A}\right)$ is the EE, $b_{k}$ are constants of order $O\left(G^{-1}\right)$. The single interval and two intervals examples both satisfy the ansatz (3.21). One could refer to [22, 39] for more supports on the ansatz. It is out of the scope of this paper to show the ansatz is true for any geomemtric states. To approach this one needs to study more details of the solutions of Einstein equation.

\subsubsection{Density of eigenstates and microcanonical ensemble}

The density of eigenstates can be obtained by using (2.6),

$$
\mathcal{P}(t)=\frac{1}{2 \pi i} \int_{\gamma-i \infty}^{\gamma+i \infty} d n e^{s_{n}}
$$

with

$$
s_{n}=n t+n S^{\infty}+(1-n)\left(S\left(\rho_{A}\right)+\sum_{k=1}(1-n)^{k} b_{k}\right),
$$

where we have used the maximal eigenvalue $\lambda_{m}=e^{-S^{\infty}}$ and the ansatz (3.21). We will take the large $c$ limit and assume $t \sim c$. Thus we could use the saddle point approximation to evaluate the inverse Lapalce transformation. We can rewrite $s_{n}$ as

$$
s_{n}=n t+n S^{\infty}+(1-n) S\left(\rho_{A}\right)+(1-n)^{2} \sum_{k=1}(1-n)^{k-1} b_{k} .
$$

The saddle point approximation requires the solution of the equation

$$
\partial_{n} s_{n}=t+S^{\infty}-S\left(\rho_{A}\right)-2(1-n) \sum_{k=1}(1-n)^{k-1} b_{k}+(1-n)^{2} \partial_{n}\left(\sum_{k=1}(1-n)^{k-1} b_{k}\right)=0 .
$$

For general $t$ it is hard to solve the above equation even if one knows the parameters $b_{k}$. However, a special case is at the point $t_{0}=S\left(\rho_{A}\right)-S^{\infty}$, for which the solution is $n=1$. Taking the solution back to $s_{n}$, the density of eigenstates is given by

$$
\mathcal{P}\left(t_{0}\right) \simeq e^{S\left(\rho_{A}\right)}
$$


One could construct the microcanonical ensemble states $\rho_{A, m}(2.24)$ with $t=t_{0}$, the entropy of which is equal to the EE of $A$ upto the leading order of $c$. This supports that the microcanonical ensemble state with can be taken as approximate state of $\rho_{A}$.

As a check of the general result, for $T<(3-2 \sqrt{2}) R$ in section 3.1 by using (3.17) we have $S^{\infty}=b_{1}+b_{2}+\delta b^{\prime}$ and $S\left(\rho_{A_{1} A_{2}}\right)=2\left(b_{1}+b_{2}\right)$. We obtain $t_{0}=b_{1}+b_{2}-\delta b^{\prime}$ by using the results in this section, which is consistent with (3.18).

\subsubsection{Correlation functions in microcanonical ensemble state}

Generally, we will consider $\operatorname{tr}\left(\rho_{A, m} \mathcal{S}\right)$ where $\mathcal{S}$ denotes the product of local operators, i.e.,

$$
\mathcal{S}=\mathcal{O}\left(x_{1}\right) \mathcal{O}\left(x_{2}\right) \ldots \mathcal{O}\left(x_{n}\right)
$$

To get $\operatorname{tr}\left(\rho_{A, m} \mathcal{S}\right)$ one needs to know $\langle\mathcal{S}\rangle_{\mathcal{R}_{n}}$ by using (2.14). Let's denote the maximal distance between the boundary of $A$ and the set $\left\{x_{1}, \ldots, x_{n}\right\}$ to be $D_{m}$. And denoting $x_{m}$ to be the maximal distance among the set $\left\{x_{1}, \ldots, x_{n}\right\}$. If the operators of $\mathcal{S}$ is located in a small region in $A$ and far away from the boundary, that is $x_{m} \ll D_{m}$, we expect the following expansion

$$
\langle\mathcal{S}\rangle_{R_{n}}=\operatorname{tr}\left(\rho_{A} \mathcal{S}\right)+\sum_{k=1}(1-n)^{k} S_{k}
$$

where $S_{k}$ are some functions depending on $x_{1}, \ldots, x_{n}$. The expansion is consistent with the fact that $\lim _{n \rightarrow 1}\langle\mathcal{S}\rangle_{\mathcal{R}_{n}}=\operatorname{tr}\left(\rho_{A} \mathcal{S}\right)$. But we don't expect this expansion is still true for $x_{m} \sim D_{m}$. The correlation functions $\langle\mathcal{S}\rangle_{\mathcal{R}_{n}}$ on $\mathcal{R}_{n}$ is same as correlation functions with the inserting of the twist operators $\sigma_{n}$ which is located at the boundary of $A$. For $x_{m} \sim D_{m}$ some operators in $\mathcal{S}$ are near the boundary of $A$. Therefore, the operator product expansion (OPE) of $\mathcal{S}$ and $\sigma_{n}$ would give main contributions to the correlation functions. The expansion form (3.28) may broke down. Our example in section 2.2.5 can be taken as an non-trivial support on our argument. Specially, the expansion (3.28) is expected to be true for $\mathcal{S}$ being the quasi-primary operators $\mathcal{X}$ in the vacuum conformal family.

By using (2.12) and (2.13) we get the correlation functions in the microcanonical state

$$
\mathcal{P}_{\mathcal{S}}(t)=\frac{1}{2 \pi i} \int_{\gamma-i \infty}^{\gamma+i \infty} d n e^{s_{n}}\langle\mathcal{S}\rangle_{\mathcal{R}_{n}}
$$

where $s_{n}$ is given by (3.23). Since the saddle point approximation would give $n=1$ if $t=t_{0}=S\left(\rho_{A}\right)-S^{\infty}$, the only survive term in (3.28) is $\operatorname{tr}\left(\rho_{A} \mathcal{S}\right)$. This gives

$$
\mathcal{P}_{\mathcal{S}}\left(t=t_{0}\right) \simeq \mathcal{P}\left(t_{0}\right) \operatorname{tr}\left(\rho_{A} \mathcal{S}\right)
$$

or

$$
\overline{\mathcal{P}}_{\mathcal{S}}\left(t=t_{0}\right):=\frac{\mathcal{P}_{\mathcal{S}}\left(t=t_{0}\right)}{\mathcal{P}\left(t_{0}\right)} \simeq \operatorname{tr}\left(\rho_{A} \mathcal{S}\right)
$$

The physical meaning underlying the results is $\rho_{A, m}$ and $\rho_{A}$ are indistinguishable if the probes are located in a small region $\tilde{A}$ and far away from the boundary of $A$.

We may define the reduced density matrix of the small region $\tilde{A}$ as $\rho_{\tilde{A}, m}:=\operatorname{tr}_{A-\tilde{A}} \rho_{A, m}$ and $\rho_{\tilde{A}}:=\operatorname{tr}_{A-\tilde{A}} \rho_{A}$. Specially, for arbitrary states in 2D CFT one could show this statement by using the short interval expansion of relative entropy, which can be seen as a 
measure of distance between two states $\rho_{\tilde{A}, m}$ and $\rho_{\tilde{A}}$. The relative entropy can be expanded as powers of the length of interval $\ell[31,32]$. We can show any order of the expansion is associated with $\operatorname{tr}\left(\rho_{A} \mathcal{X}\right)-\operatorname{tr}\left(\rho_{A, m} \mathcal{X}\right)$, where $\mathcal{X}$ denotes the operators contained in the theory. Our result (3.31) shows the relative entropy is vanishing to any order of $\ell$ in the order of $O(c)$. By using the Pinsker's inequality one can show the trace distance between the two states are also zero .

Of course, one can always find the probes to distinguish the two states, for example, the Rényi entropies of $\rho_{A}$ and $\rho_{A, m}$ are different. This shows their difference will appear if the probes are not limited to $\tilde{A}$.

\subsection{Higher dimensions}

The set-up in previous sections can be easily generalized to higher dimensions case. Once the Rényi entropies $S_{A}^{(n)}$ are known, one could evaluate the density of eigenstates by using (2.6). For the holographic CFTs the Rényi entropy of a sphere are calculated in [22]. For d-dimensional CFTs dual to Einstein gravity the Rényi entropy of a sphere in the vacuum state is given by

$$
S_{A}^{(n)}(d)=\frac{n}{8 G(n-1)} V_{\Sigma}\left(2-x_{n}^{d-2}-x_{n}^{d}\right),
$$

where we take the radius of AdS $L=1, x_{n}=\frac{1}{n d}\left(1+\sqrt{1-2 d n^{2}+d^{2} n^{2}}\right), V_{\Sigma}$ is a universal term depending on dimension $d$

$$
V_{\Sigma}(d)= \begin{cases}(-1)^{d / 2-1} \frac{2}{\pi} \log (2 R / \epsilon) & \text { for even d } \\ (-1)^{(d-1) / 2} & \text { for odd d. }\end{cases}
$$

We have the $\operatorname{EE} S\left(\rho_{A}\right)=\lim _{n \rightarrow 1} S_{A}^{(n)}(d)=\frac{1}{4 G} V_{\Sigma}$. Taking the above result into (2.6) we get the density of eigenstates $\mathcal{P}(t)$. According to the discussions in section 3.2.2 we only need to show the Rényi entropies satisfy the general ansatz (3.21). In the region $n \sim 1$ we have the expansion

$$
S_{A}^{(n)}(d)=\frac{1}{4 G} V_{\Sigma}-\frac{n-1}{8 G}+\frac{\left(4 d^{2}-8 d+3\right)(n-1)^{2}}{24(d-1)^{2} G}+O\left((n-1)^{3}\right),
$$

which is consistent with the ansatz (3.21). Therefore, using the result in section 3.2.2 we have

$$
\mathcal{P}\left(t=t_{0}\right) \simeq e^{S\left(\rho_{A}\right)},
$$

for $t_{0}=S\left(\rho_{A}\right)-S^{\infty}$, where

$$
S^{\infty}:=\lim _{n \rightarrow \infty} S_{A}^{(n)}(d)=\frac{(1-d)\left(\frac{\sqrt{(d-2) d}}{d}\right)^{d}+d-2}{4(d-2) G} V_{\Sigma} .
$$

In the appendix $\mathrm{C}$ we show this result by direct calculation for $d=4$.

To get the correlation functions of the local operators (3.27) in the mircocanonical ensemble state with $t=t_{0}$ one needs to know the correlation functions on $\mathcal{R}_{n}$. In [40] the 
authors studied the twist operators in higher dimensions. The one-point functions of stress energy tensor on $\mathcal{R}_{n}$ are evaluated in that paper. The results are

$$
\left\langle T_{\mu \nu}\right\rangle_{\mathcal{R}_{n}}=\left\langle T_{\mu \nu} \sigma_{n}\right\rangle \propto h_{n}
$$

where $\sigma_{n}$ is the higher dimensional twist operator, $h_{n}$ is the dimension of the twist operator. An important result of [40] is

$$
h_{n}=\sum_{k=1}^{\infty} \frac{1}{k !} H_{k}(n-1)^{k}
$$

where $H_{k}$ are constants independent with $n$. Thus, the one-point functions $\left\langle T_{\mu \nu}\right\rangle_{\mathcal{R}_{n}}$ satisfy the ansatz (3.28) with $\left\langle T_{\mu \nu}\right\rangle=0$ by symmetry of stress energy tensor on the infinite plane. Therefore, we will have

$$
\overline{\mathcal{P}}_{T_{\mu \nu}}\left(t=t_{0}\right): \simeq\left\langle T_{\mu \nu}\right\rangle=0,
$$

where $\overline{\mathcal{P}}_{T_{\mu \nu}}(t)$ is defined as (3.31) with $\mathcal{S}=T_{\mu \nu}$. This results support the idea that the microcanonical ensemble state with $t=t_{0}$ can be taken as an approximated state of $\rho_{A}$ in the higher dimensions.

\section{Applications of the microcanonical ensemble states}

In previous sections we discuss the microcanonical ensemble state $\rho_{A, m}$. The number of the microstates in the ensemble is large which is of order $O\left(e^{c}\right)$. In this section we will come back to (1.5) and find its constraints on the eigenstates and eigenvalues of $\rho_{A}$.

\subsection{Holevo information}

Let's consider the subsystem $A=A_{1} A_{2} A_{3}$ in the vacuum state of 2D CFTs. We have the reduced density matrix $\rho_{A_{1} A_{2}}$ and $\rho_{A_{3}}$,

$$
\rho_{A_{1} A_{2}}=\sum_{i} \lambda_{i} \rho_{i, A_{1} A_{2}}, \quad \rho_{A_{3}}=\sum_{i} \lambda_{i} \rho_{i, A_{3}},
$$

where $\rho_{i, A_{1} A_{2}}:=\operatorname{tr}_{A_{3}}\left|\lambda_{i}\right\rangle_{A}{ }_{A}\left\langle\lambda_{i}\right|$ and $\rho_{i, A_{3}}:=\operatorname{tr}_{A_{1} A_{2}}\left|\lambda_{i}\right\rangle_{A}{ }_{A}\left\langle\lambda_{i}\right|$. For an ensemble of a mixed state $\rho=\sum_{i} p_{i} \rho_{i}$, we may define the Holevo information,

$$
\chi(\rho):=S(\rho)-\sum_{i} p_{i} S\left(\rho_{i}\right) .
$$

The Holevo information is an upper bound of the information that one can gain from the ensemble. It can also be used to characterize the distinguishability between the microstates $\rho_{i}$ [41]. It is obvious that the Holevo information is non-negative, i.e., $\chi(\rho) \geq 0$. It can also be shown that $\chi(\rho) \leq H\left(p_{i}\right)=-\sum_{i} p_{i} \log p_{i}$. We are interested in the condition (1.5), which gives constraints on the spectrum and eigenstate of $\rho_{A_{1} A_{2} A_{3}}$. We may write (1.5) as

$$
\chi\left(\rho_{A_{1} A_{2}}\right)=\chi\left(\rho_{A_{3}}\right)+H\left(\lambda_{i}\right),
$$


where $H\left(\lambda_{i}\right)=-\sum_{i} \lambda_{i} \log \lambda_{i}, \rho_{A_{1} A_{2}}$ and $\rho_{A_{3}}$ are given by the ensembles (4.1). In the above derivation we use the fact $S\left(\rho_{i, A_{3}}\right)=S\left(\rho_{i, A_{1} A_{2}}\right)$. Using the bound of the Holevo information we have

$$
\chi\left(\rho_{A_{1} A_{2}}\right)=H\left(\lambda_{i}\right) \quad \text { and } \quad \chi\left(\rho_{A_{3}}\right)=0 .
$$

This means the microstates $\left\{\rho_{i, A_{3}}\right\}$ are indistinguishable from $\rho_{A_{3}}$, while $\left\{\rho_{i, A_{1} A_{2}}\right\}$ can be perfectly distinguished.

The RT formula (1.1) and the relation (1.5) only count the leading order contribution in the limit $G \rightarrow 0$. In the CFT this corresponds to the limit $c \rightarrow \infty$ with $c \sim 1 / G$. The EE should have quantum corrections which is of order $O\left(c^{0}\right)$. Therefore, the results should include higher order corrections of large $c$. More precisely. we have

$$
\chi\left(\rho_{A_{1} A_{2}}\right)=H\left(\lambda_{i}\right)+O\left(c^{0}\right) \quad \text { and } \quad \chi\left(\rho_{A_{3}}\right)=O\left(c^{0}\right) .
$$

\subsection{Operational meaning of the Holevo information}

We should stress that the formulas of Holevo information (4.5) is equal to (1.5). Holevo information has an operational meaning that is an upper bound of the accessible information from a given ensemble. The accessible information is associated with the operations or measurements on the ensemble. Generally, the measurement can be described by the so-called Positive Operator-Valued Measure (POVM) elements. The measurement includes the positive operators

$$
E_{k}:=M_{k}^{\dagger} M_{k}
$$

which satisfy the completeness condition $\sum_{k} E_{k}=I$. For the measurement on a state $\rho$ the probability of outcome $k$ is given by $p_{k}=\operatorname{tr}\left(\rho E_{k}\right)$. The state after measurement is

$$
\rho_{k}=\frac{M_{k} \rho M_{k}^{\dagger}}{\operatorname{tr}\left(M_{k}^{\dagger} M_{k} \rho\right)} .
$$

In the view of algebraic quantum field theories the POVM elements belong to the observable algebra $\mathcal{U}(A)$ associated with a region $A$.

For a given ensemble $\rho=\sum_{i \in X} p_{i} \rho_{i}$ we can take the index $X=\{1,2, \ldots\}$ as a random variable with the probability $\left\{p_{1}, p_{2}, \ldots\right\}$. With the measurement $E_{Y}=\left\{E_{1}, E_{2}, \ldots\right\}$ the outcomes $Y$ denote the other random variable. We have the condition probability $p(y \mid x)=$ $\operatorname{tr}\left(\rho_{x} E_{y}\right)$ and the joint distribution $p_{x, y}=p_{x} p(y \mid x)$. The accessible information is given by the mutual information $I(X, Y)=H\left(p_{x}\right)+H\left(p_{y}\right)-H\left(p_{x, y}\right)$ where $p_{x}:=\sum_{j \in Y} p_{x, y}$ and $p_{y}:=\sum_{i \in X} p_{x, y}$. The Holevo information is a bound of the accessible information, i.e., $I(X, Y) \leq \chi(\rho)$ with $\rho=\sum_{i \in X} p_{i} \rho_{i}$. With some calculations we have

$$
H(X, Y)=\sum_{i \in X, j \in Y} p_{i} \operatorname{tr}\left(\rho_{i} E_{j}\right) \log \frac{\operatorname{tr}\left(\rho_{i} E_{j}\right)}{\operatorname{tr}\left(\rho E_{j}\right)}
$$

Note that $H(X, Y)=0$ if and only if $\operatorname{tr}\left(\rho_{i} E_{j}\right)=\operatorname{tr}\left(\rho E_{j}\right)$ for any $i \in X$ and $j \in Y$ that means any measurement cannot distinguish the states $\rho_{i}(i \in X)$. 


\subsection{Holevo information $\chi\left(\rho_{A_{3}}\right)$}

Now let's turn to the reduced density matrix of a single interval in the vacuum state $\rho_{A}=\sum_{i} \lambda_{i}|i\rangle_{A}{ }_{A}\langle i|$. Support the measurements are located in a small region, say $A_{3}$ with $T \ll R$, which means $E_{j} \in \mathcal{U}\left(A_{3}\right)(j \in Y)$. The mutual information $H(X, Y)$ in the state $\rho_{A}$ is given by

$$
\left.H(X, Y)\right|_{\rho_{A}}=\sum_{i, j} \lambda_{i}\left\langle E_{j}\right\rangle_{i} \log \left\langle E_{j}\right\rangle_{i}-\sum_{j}\left\langle E_{j}\right\rangle_{\rho_{A}} \log \left\langle E_{j}\right\rangle_{\rho_{A}},
$$

where $\left\langle E_{j}\right\rangle_{i}:={ }_{A}\left\langle i\left|E_{j}\right| i\right\rangle_{A}$ and $\left\langle E_{j}\right\rangle_{\rho_{A}}:=\operatorname{tr}\left(\rho_{A} E_{j}\right) .\left.H(X, Y)\right|_{\rho_{A}}$ is equal to the one defined by the state $\rho_{A_{3}}=\sum_{i} \lambda_{i} \rho_{i, A_{3}}$ since ${ }_{A}\left\langle i\left|E_{j}\right| i\right\rangle_{A}=\operatorname{tr}\left(\rho_{i, A_{3}} E_{j}\right)$. The POVM elements $E_{j}$ are bounded operators which are composed by the local operators $\mathcal{O}(x)$ with $x \in A_{3}$. In section 3.2.3 we have shown the expectation value of the products of the local operators $\mathcal{S}$ (3.27) in the mircocanonical states with $t=t_{0}$ would approach to $\operatorname{tr}\left(\rho_{A} \mathcal{S}\right)$ in the large $c$ limit. Therefore, if $T \ll R$ we expect $\operatorname{tr}\left(\rho_{A, m} E_{j}\right) \rightarrow \operatorname{tr}\left(\rho_{A} E_{j}\right)$ in the large $c$ limit. The first term of (4.9) can be written as

$$
\sum_{i, j} \lambda_{i}\left\langle E_{j}\right\rangle_{i} \log \left\langle E_{j}\right\rangle_{i}=\sum_{j} \int_{0}^{+\infty} d t\left(\mathcal{P}(t) \lambda_{m} e^{-t}\right) \overline{\mathcal{P}}_{E_{j}}
$$

where

$$
\overline{\mathcal{P}}_{E_{j}}:=\frac{\sum_{i}\left\langle E_{j}\right\rangle_{i} \log \left\langle E_{j}\right\rangle_{i} \delta\left(t_{i}-t\right)}{\mathcal{P}(t)} .
$$

By using (2.23) for $t \neq 0$ we have

$$
\mathcal{P}(t) \lambda_{m} e^{-t}=\frac{b e^{-(\sqrt{b}-\sqrt{t})^{2}}}{\sqrt{4 \pi}(b t)^{3 / 4}} \rightarrow \delta(t-b)
$$

in the limit $b \rightarrow \infty$ or $c \rightarrow \infty$. Therefore, we have

$$
\sum_{i, j} \lambda_{i}\left\langle E_{j}\right\rangle_{i} \log \left\langle E_{j}\right\rangle_{i} \rightarrow \sum_{j} \frac{1}{\mathcal{P}(b)} \sum_{i}\left\langle E_{j}\right\rangle_{i} \log \left\langle E_{j}\right\rangle_{i} \delta\left(t_{i}-b\right) .
$$

Combination of the above results we find

$$
\left.\left.H(X, Y)\right|_{\rho_{A}} \rightarrow H(X, Y)\right|_{\rho_{A, m}},
$$

in the large $c$ or $b$ limit. One could define the Holevo information of $\rho_{A_{3}, m}:=\frac{1}{\mathcal{P}(b)} \sum_{k} \delta\left(t_{i}-\right.$ b) $\rho_{i, A_{3}}$ as $\chi\left(\rho_{A_{3}, m}\right)$. We conclude if $T \ll R$

$$
\chi\left(\rho_{A_{3}}\right)=\chi\left(\rho_{A_{3}, m}\right),
$$

in the large $c$ limit. This equality gives us a way to explain the almost vanishing Holevo information of $\chi\left(\rho_{A_{3}}\right)$ in the case $T<(3-2 \sqrt{2}) R$. At present we only consider $T \ll R$. The mutual information $\left.H(X, Y)\right|_{\rho_{A_{3}, m}}=\left.H(X, Y)\right|_{\rho_{A, m}}$ is

$$
\left.H(X, Y)\right|_{\rho_{A_{3}, m}}=\frac{1}{\mathcal{P}(b)} \sum_{i, j}\left\langle E_{j}\right\rangle_{i} \log \frac{\left\langle E_{j}\right\rangle_{i}}{\left\langle E_{j}\right\rangle_{\rho_{A, m}}} \delta\left(t_{i}-b\right) .
$$


Let's denote $e_{i j}:=\frac{\left\langle E_{j}\right\rangle_{i}}{\left\langle E_{j}\right\rangle_{\rho_{A, m}}}$. Since $\frac{1}{\mathcal{P}(b)} \sum_{i} e_{i j} \delta\left(t_{i}-b\right)=1$ and $\left\langle E_{j}\right\rangle_{i} \geq 0$, it is obvious $e_{\max }=\max _{i, j} e_{i j} \geq 1$ and $e_{\min }=\min _{i, j} e_{i j} \leq 1$. The microstates in $\rho_{A_{3}, m}$ are eigenstates of $H_{A}$ with the same eigenvalues. A natural assumption is that the measurements $E_{j}$ cannot distinguish the microstates $|i\rangle_{A}$ at the leading order of $c$. For most of tensor $e_{i j}{ }^{1}$ we expect the expansion

$$
\log e_{i j}=\tilde{e}_{i j}+O(1 / c)
$$

where $\tilde{e}_{i j}$ are of order $O\left(c^{0}\right)$. With the ansatz we have

$$
\left.H(X, Y)\right|_{\rho_{A_{3}, m}} \simeq \frac{1}{\mathcal{P}(b)} \sum_{i, j}\left\langle E_{j}\right\rangle_{i} \tilde{e}_{i j}
$$

Support $\tilde{e}_{\max }:=\max _{i j}\left|\tilde{e}_{i j}\right|$. We have

$$
\left.H(X, Y)\right|_{\rho_{A_{3}, m}} \leq \tilde{e}_{\max }
$$

This result is consistent with $\chi\left(\rho_{A_{3}}\right)=O\left(c^{0}\right)$.

\subsection{Constraints on one-point functions of quasi-primary operators}

One could calculate the EE and Holevo information for arbitrary state $\rho=\sum_{i} \lambda_{i} \rho_{i}$ directly by using the short interval expansion method. In the appendix $\mathrm{D}$ we review the short expansion method. The Holevo information $\chi\left(\rho_{A}\right)$ is associated with the terms of the form

$$
\left\langle\mathcal{X}_{1}\right\rangle_{\rho} \ldots\left\langle\mathcal{X}_{m}\right\rangle_{\rho}-\sum_{i} p_{i}\left\langle\mathcal{X}_{1}\right\rangle_{i}\left\langle\mathcal{X}_{m}\right\rangle_{i}
$$

where $\langle\mathcal{X}\rangle_{i}:=\operatorname{tr}\left(\rho_{i} \mathcal{X}\right)$. Support the length of the interval is $\ell$. Let's consider the microcanonical ensemble state $\rho_{A, m}$. We have shown in section 2.2.3 the one-point function $\operatorname{tr}\left(\rho_{A, m} \mathcal{X}\right) \rightarrow 0$ in the large $c$ limit. Therefore, $\chi\left(\rho_{A, m}\right)$ only depends on the $\langle\mathcal{X}\rangle_{i}:={ }_{A}\left\langle\lambda_{i}|\mathcal{X}| \lambda_{i}\right\rangle_{A}$ with $\mathcal{X}=T, \mathcal{A}$ and their derivatives upto $O\left(\ell^{10}\right)$.

We have calculated the average one-point functions of $T$ and $\mathcal{A}$ in the microcanonical ensemble state $\rho_{A, m}$ in section 2.2.2. Let's define the functions

$$
\begin{aligned}
& \mathrm{t}\left(w ; \lambda_{i}\right):={ }_{A}\left\langle\lambda_{i}|T(w)| \lambda_{i}\right\rangle_{A}-\overline{\mathcal{P}}_{T}\left(t_{i}\right), \\
& \mathrm{a}\left(w ; \lambda_{i}\right):={ }_{A}\left\langle\lambda_{i}|\mathcal{A}(w)| \lambda_{i}\right\rangle_{A}-\overline{\mathcal{P}}_{\mathcal{A}}\left(t_{i}\right) .
\end{aligned}
$$

By definition we have

$$
\frac{1}{\mathcal{P}(t)} \sum_{i} \mathrm{t}\left(w ; \lambda_{i}\right) \delta\left(t_{i}-t\right)=0, \quad \frac{1}{\mathcal{P}(t)} \sum_{i} \mathrm{a}\left(w ; \lambda_{i}\right) \delta\left(t_{i}-t\right)=0 .
$$

By using the results (2.41) we have

$$
\int_{-R+\epsilon}^{R-\epsilon} d w \frac{R^{2}-w^{2}}{2 R}{ }_{A}\left\langle\lambda_{i}|T(w)| \lambda_{i}\right\rangle_{A}=\frac{1}{2}(b-t) .
$$

\footnotetext{
${ }^{1}$ Here we don't need all of the tensor $e_{i j}$ satisfy (4.17). Assume there exists some states with number $N$ such that $e_{i j} \sim c$. As long as the number $N \ll \mathcal{P}(b) \sim O\left(e^{c}\right)$ their contributions to (4.16) are exponentially suppressed.
} 
This gives

$$
\int_{-R+\epsilon}^{R+\epsilon} d w \frac{R^{2}-w^{2}}{2 R} \mathrm{t}\left(w ; \lambda_{i}\right)=0 .
$$

In general, we can expand $\mathrm{t}\left(x ; \lambda_{i}\right)$ by the Legendre Polynomials as follows ${ }^{2}$

$$
\mathrm{t}\left(w ; \lambda_{i}\right)=\sum_{j \geq 3} c_{i j} P_{j}\left(\frac{w}{R}\right)
$$

where $c_{i j}$ are constants independent with $w$. By using the short interval expansion of Holevo information (D.3) and the condition $\chi\left(\rho_{A, m}\right)=O\left(c^{0}\right)$, we find the constraints on $\mathrm{t}\left(w ; \lambda_{i}\right)$ at order of $O\left(\ell^{4}\right)$,

$$
\frac{1}{\mathcal{P}(b)} \sum_{i} \mathrm{t}\left(w ; \lambda_{i}\right)^{2} \delta\left(t_{i}-b\right) \lesssim O(c)
$$

where $\lesssim$ means the left hand side is at most of order $O(c)$. A natural assumption is that most of the functions $\mathrm{t}\left(w ; \lambda_{i}\right)$ are at most of order $O(\sqrt{c})$. One may define the maximal value of $\mathrm{t}\left(w ; \lambda_{i}\right)$ among these functions as $\mathrm{t}(w)_{\max }:=\max _{\lambda_{i}}\left|\mathrm{t}\left(w ; \lambda_{i}\right)\right|$. If $\mathrm{t}(w)_{\max } \lesssim O(\sqrt{c})$, we would obtain (4.26).

Taking the large $c$ limit, the term at order of $O\left(\ell^{8}\right)$ becomes

$$
\frac{1}{630 c^{2}} \frac{1}{\mathcal{P}(b)} \sum_{i}\left(\mathrm{a}\left(w ; \lambda_{i}\right)-\mathrm{t}\left(w ; \lambda_{i}\right)^{2}\right)^{2} \delta\left(t_{i}-b\right) .
$$

By the similar argument as above we conclude most of a $\left.\left(w ; \lambda_{i}\right)\right|_{\lambda_{i}=\lambda_{0}}$ should be at most of order $O(c)$. One may define $\mathrm{a}(w)_{\max }:=\max _{\lambda_{i}}\left|\mathrm{a}\left(w ; \lambda_{i}\right)\right|$. The assumption $\mathrm{a}(w)_{\max } \lesssim O(c)$ ensures $\chi\left(\rho_{A, m}\right) \lesssim O\left(c^{0}\right)$ upto order $O\left(\ell^{8}\right)$.

One could check the term of Holevo information $\chi\left(\rho_{A, m}\right) \lesssim O\left(c^{0}\right)$ upto $O\left(\ell^{10}\right)$ if $\mathrm{t}(w)_{\max } \lesssim O(\sqrt{c})$ and $\mathrm{a}(w)_{\max } \lesssim O(c)$ are satisfied.

\section{Conclusions and discussions}

In the context of AdS/CFT the geometric states should be the ones that are very special. They would show some well-defined and special properties in the semi-classical limit $G \rightarrow 0$ or $c \rightarrow \infty$. In this paper we focus on the entanglement spectrum of the geometric states, which contain more information of the reduced density matrix $\rho_{A}$ than the EE. We can use the inverse Laplace transformation with respect to the index $n$ of Rényi entropy.

A single interval $A$ in the vacuum state of $2 \mathrm{D}$ CFTs is the example that one can exactly get the eigenstates and eigenvalues of $\rho_{A}$. By direct calculation we show there exists a mircocanonical ensemble states $\rho_{A, m}$ with $t_{0}=-\log \lambda_{m}$ can be taken as an approximate state of $\rho_{A}$ in the large $c$ limit if our probes are located in a small region of $A$ and far away from the boundary. We get the conclusion by evaluating the one-point functions of primary and quasi-primary operators and two-point functions of primary operator in the mircocanonical ensemble state. The one-point functions are always consistent with the

\footnotetext{
${ }^{2}$ We can ignore $\epsilon$ in the integration.
} 
one in $\rho_{A}$, that is vanishing. The two-point functions are consistent only if the distance between two operators are small. We should stress that the results are only true in the semi-classical limit $c \rightarrow \infty$.

For the two intervals example we evaluate the micrcocanonical ensemble state with $t_{0}$ (3.18) by using saddle point approximation. The parameter $b_{0}$ is not only associated with the length of subsystem $A_{1}$ and $A_{2}$, but also related to the cross ratio of the two intervals at the order of $O(c)$. Let's comment on the two point correlation functions

$$
\mathcal{P}_{\mathcal{O}_{A_{1}} \mathcal{O}_{A_{2}}}\left(t=t_{0}\right)=\sum_{J}\left\langle\Lambda_{J}\left|\mathcal{O}_{A_{1}} \mathcal{O}_{A_{2}}\right| \Lambda_{J}\right\rangle \delta\left(t_{J}-t_{0}\right)
$$

where $\mathcal{O}_{A_{1}\left(A_{2}\right)}$ are operators in the region $A_{1}\left(A_{2}\right)$. Or the normalized ones

$$
\overline{\mathcal{P}}_{\mathcal{O}_{A_{1}} \mathcal{O}_{A_{2}}}\left(t=t_{0}\right):=\frac{\mathcal{P}_{\mathcal{O}_{A_{1}} \mathcal{O}_{A_{2}}}\left(t=t_{0}\right)}{\mathcal{P}\left(t=t_{0}\right)},
$$

where $\mathcal{P}\left(t=t_{0}\right)$ is given by (3.14). To get the two point functions one needs

$$
\left\langle\mathcal{O}_{A_{1}} \mathcal{O}_{A_{2}}\right\rangle_{\mathcal{R}_{n}}=\left\langle\mathcal{O}_{A_{1}} \mathcal{O}_{A_{2}} \sigma_{n}(-R) \sigma_{n}(-T) \sigma_{n}(T) \sigma_{n}(R)\right\rangle,
$$

where $\sigma_{n}$ are the twist operators which are local in 2D CFTs, the correlator on r.h.s. is evaluated on n-copied CFTs. Let's consider the limit $\eta \ll 1$, which means the distance between $A_{1}$ and $A_{2}$ is far larger than their own sizes. By the OPE of CFTs we have

$$
\left\langle\mathcal{O}_{A_{1}} \mathcal{O}_{A_{2}}\right\rangle_{\mathcal{R}_{n}} \simeq\left\langle\mathcal{O}_{A_{1}} \sigma_{n}(-R) \sigma_{n}(-T)\right\rangle\left\langle\mathcal{O}_{A_{2}} \sigma_{n}(T) \sigma_{n}(R)\right\rangle=\left\langle\mathcal{O}_{A_{1}}\right\rangle_{\mathcal{R}_{n}}\left\langle\mathcal{O}_{A_{2}}\right\rangle_{\mathcal{R}_{n}}
$$

The above result doesn't satisfy the ansatz (3.28). Therefore, we expect the two point functions $\overline{\mathcal{P}}_{\mathcal{O}_{A_{1}} \mathcal{O}_{A_{2}}}\left(t=t_{0}\right)$ are different from $\operatorname{tr}\left(\rho_{A_{1} A_{2}} \mathcal{O}_{A_{1}} \mathcal{O}_{A_{2}}\right)=\left\langle\mathcal{O}_{A_{1}} \mathcal{O}_{A_{2}}\right\rangle$. This expectation is also consistent with our arguments in section 3.2.3. Here the distance between two operators $\mathcal{O}_{A_{1}}, \mathcal{O}_{A_{2}}$ are larger than their distances to the boundary.

By using the proposal of holographic Rényi entropy, we generalize the results to arbitrary geometric states. The key point is that the holographic Rényi entropy is that $S^{(n)} \sim O(c)$. This permits us to use the saddle point approximation to find the microcanonical ensemble state $\rho_{A, m}$ with the parameter $t_{0}$. The solution of the saddle point approximation gives $n=1$. The parameter $t_{0}$ has a simple expression $t_{0}=S\left(\rho_{A}\right)-S^{\infty}$, where $S\left(\rho_{A}\right)$ is the EE and $S^{\infty}$ is $\lim _{n \rightarrow \infty} S^{(n)}$. The entropy of the microcanonical ensemble state $\rho_{A, m}$ with $t_{0}$ is equal to the EE of $A$. However, it cannot give the same Rényi entropy of $A$. The reason is that the microcanonical ensemble state can only be an approximate state of $\rho_{A}$ only if the probes are located in a small region of $A$ and far away from the boundary of $A$. This is consistent with the example of a single interval in the vacuum state. We check this by comparing $\operatorname{tr}\left(\rho_{A, m}\right) \mathcal{S}$ with $\operatorname{tr}\left(\rho_{A} \mathcal{S}\right)$, where $\mathcal{S}(3.27)$ is product of the local operators. A remarkable result is that $\operatorname{tr}\left(\rho_{A, m}\right) \mathcal{S} \rightarrow \operatorname{tr}\left(\rho_{A} \mathcal{S}\right)$ if $\mathcal{S}$ permits the expansion as (3.28), which is expected to be true if the local operators are located in a small region in $A$ and far away from the boundary.

Finally, we discuss the equality condition of the Araki-Lieb inequality (1.5). The condition can be reformed as the Holevo information, which can be taken as an upper bound 
of information that one can gain by arbitrary measurements. To satisfy this condition we find the constraints on the expectation values of measurements and local operators. This constraints would help us to understand more on properties of geometric states.

In the following we will discuss some unsolved problems that are worth to explore in the future.

\subsection{Transition between distinguishability and indistinguishability}

As mentioned above we expect $\rho_{A, m}$ and $\rho_{A}$ are indistinguishable at the leading order of $c$ only if the probes are located in a small region. If using the Rényi entropy or two-point correlation functions with large distance, one would find the difference between the two states. However, we cannot find the critical point where the transition between distinguishability and indistinguishability happens. Let's see the two-point functions (2.51) in the microcanonical ensemble $\rho_{A, m}$. The average two-point function $\overline{\mathcal{P}}_{\mathcal{O O}}(t) \rightarrow \operatorname{tr}\left(\rho_{A} \mathcal{O O}\right)$ if $t=b$ upto $O\left(x^{6}\right)$. But when the distance $2 x$ between two operators is large enough, the perturbative expansion with respect to $x$ may broke. The difference between $\rho_{A}$ and $\rho_{A, m}$ with $t=b$ will appear.

An interesting question is whether the transition between distinguishability and indistinguishability is associated with the critical point $T=(3-2 \sqrt{2}) R$. For $T<(3-2 \sqrt{2}) R$ we have $\chi\left(\rho_{A_{3}}\right)=O\left(c^{0}\right)$. For $T>(3-2 \sqrt{2}) R$ we expect $\chi\left(\rho_{A_{3}}\right) \sim O(c)$. In section 4.3 we show the $\chi\left(\rho_{A_{3}}\right) \simeq \chi\left(\rho_{A_{3}, m}\right)$ with $\mathrm{t}=\mathrm{b}$ if the length of $A_{3}$ is small enough. $\chi\left(\rho_{A_{3}, m}\right)=O\left(c^{0}\right)$ means the microstates $\rho_{A_{3}, i}$ of the microcanoncial ensemble states are indistinguished at the leading order of $c$, that is the expression (4.17). In section 4.4 we directly calculate the Holevo information of $\rho_{A_{3}, m}$ by using short interval expansion. The almost vanishing $\chi\left(\rho_{A_{3}, m}\right)=O\left(c^{0}\right)$ gives $(4.21)$ with $\mathrm{t}\left(w ; \lambda_{i}\right) \lesssim O(\sqrt{c})$ and $\mathrm{a}\left(w ; \lambda_{i}\right) \lesssim O(c)$. This means the difference of the expectation values of $\mathcal{X}$ in the mircrostates $\left|\lambda_{i}\right\rangle_{A}$ of $\rho_{A, m}$ is equal to $\operatorname{tr}\left(\rho_{A, m} \mathcal{X}\right)$ in the leading order of $c$, which are natural assumptions. Our opinion is that $\chi\left(\rho_{A_{3}, m}\right)=O\left(c^{0}\right)$ is a consequence of the indistinguishability of $\rho_{A_{3}}$ from $\rho_{A_{3}, m}$. However, we cannot prove this or disprove this point at present. We will leave this to future works.

\subsection{A possible geometric explanation of Holevo information}

If a quantity can be associated with a geometric object in the bulk, we call it a geometric probe, for example the EE or Rényi entropy. To find these geometric probes are important since they would help us to understand more on the properties of geometric states. A natural requirement of the geometric quantity is that they should be order of $O(c)$. In section 4.1 we have shown the relation of holographic EE $(1.5)$ in the case $T \leq(3-2 \sqrt{2}) R$ is equal to the conditions of Holevo information (4.5). $\chi\left(\rho_{A_{3}}\right)$ is vanishing at the order of $O(c)$. However, if $T>(3-2 \sqrt{2}) R$, we expect $\chi\left(\rho_{A_{3}}\right)$ should be the order of $O(c)$. For $T \sim R$ we will have $\chi\left(\rho_{A_{3}}\right) \simeq S\left(\rho_{A}\right) \sim O(c)$. As shown in [42] the Holevo information is monotonically increasing, that is if $\rho_{X}=\operatorname{tr}_{Y} \rho_{X Y}$ we have $\chi\left(\rho_{X}\right) \leq \chi\left(\rho_{X Y}\right)$. This leads to

$$
\partial_{T} \chi\left(\rho_{A_{3}}\right) \geq 0 \text {. }
$$

Therefore, we expect $\chi\left(\rho_{A_{3}}\right)$ should be a quantity that is order of $O(c)$ and monotonically increasing with $T$ in the case $T>(3-2 \sqrt{2}) R$. We should note that $\chi\left(\rho_{A_{3}}\right)$ is not only 
dependent with the reduced density state $\rho_{A_{3}}$, but also on the spectrum decomposition of $\rho_{A}$. This is because the Holevo information depends on the ensemble. Generally, a mixed state $\rho$ can be written as different ensembles, say $\rho=\sum_{i} p_{i} \rho_{i}$ and $\rho=\sum_{j} q_{j} \rho_{j}^{\prime}$. The corresponding Holevo information $\chi\left(\sum_{i} p_{i} \rho_{i}\right)$ is different from $\chi\left(\sum_{j} q_{j} \rho_{j}^{\prime}\right)$ in general. Our discussion is based on the fixed ensemble $\rho_{A}=\sum_{i} \lambda_{i}\left|\lambda_{i}\right\rangle_{A}{ }_{A}\left\langle\lambda_{i}\right|$. Therefore, the Holevo information $\chi\left(\rho_{A_{3}}\right)$ should depend on the eigenvalues and eigenstates of $\rho_{A}$. In some sense $\chi\left(\rho_{A_{3}}\right)$ contain more information of the state $\rho_{A}$ than the EE, which is only the trace of the eigenvalues. According to the so-called subregion/subregion duality in AdS/CFT [43][46], the bulk region surround by the RT surface $\gamma_{A}$ and the boundary region $A$, named entanglement wedge, is expected to be dual to the reduced density matrix $\rho_{A} \cdot \chi\left(\rho_{A_{3}}\right)$ and $\chi\left(\rho_{A_{1} A_{2}}\right)$ do extract some information of $\rho_{A}$. If $\chi\left(\rho_{A_{3}}\right)$ has the geometric dual, then the geoemtric object should be in the region inside the entanglement wedge.

However, to get the exact dual of one quantity one should be able to evaluate the quantity both in bulk and the boundary CFTs. One could also make a guess by comparing the properties on both sides like the one that is done in the proposal of holographic entanglement of purification $[47,48]$. In the paper we are not trying to conjecture the duality of $\chi\left(\rho_{A_{3}}\right)$.

Let's comment more on the ensemble dependence of $\chi\left(\rho_{A}\right)$. In [49] we show the $\chi\left(\rho_{A_{3}}\right)=O\left(c^{0}\right)$ (by the notation of the present paper) for the ensemble of $\rho_{A_{3}}$ that seems not related to the one we used here. In that paper we consider the ensemble $\rho_{A}=\sum_{k} p_{k}|\psi\rangle_{k}{ }_{k}\langle\psi|$ that would make the entropy $\sum_{k} p_{k} S\left(\tilde{\rho}_{k, A_{3}}\right)$ minimal, where $\tilde{\rho}_{k, A_{3}}:=\operatorname{tr}_{A_{1} A_{2}}|\psi\rangle_{k}{ }_{k}\langle\psi|$. The entropy actually is defined as the entanglement of formation (EoF), which characterizes the correlation between $A_{1}$ and $A_{2}$. By using the Koashi-Winter relation we find that $\chi\left(\sum_{k} p_{k} \tilde{\rho}_{k, A_{3}}\right)$ is also the order of $O\left(c^{0}\right)$. It is interesting to study the relation between the ensemble $\rho_{A}=\sum_{k} p_{k}|\psi\rangle_{k}{ }_{k}\langle\psi|$ and the spectrum decomposition (1.2) in the near future.

\subsection{On construction of new geometric states}

We only focus on the microcanonical ensemble state $\rho_{A, m}$ with $t=t_{0}=S\left(\rho_{A}\right)-S^{\infty}$. We could construct a pure state

$$
|\psi\rangle_{0}=\frac{1}{\sqrt{\mathcal{P}\left(t_{0}\right)}} \sum_{i}\left|\lambda_{i}\right\rangle_{A} \otimes\left|\bar{\lambda}_{i}\right\rangle_{\bar{A}} \delta\left(t_{i}-t_{0}\right) .
$$

Of courese, the state $|\psi\rangle_{0}$ is different from the vacuum state. In $[50,51]$ the authors constructed an approximate tensor networks for geometric states in AdS/CFT, which is generally given by

$$
|\Psi\rangle=\sum_{I=0}^{e^{O(\sqrt{S})}} \sum_{i=0}^{e^{S-\sqrt{S}}} \sqrt{\lambda_{i}}|I, i\rangle_{A} \otimes|I, i\rangle_{\bar{A}},
$$

where $S:=S\left(\rho_{A}\right)$ is the EE of $A$. It seems our state $|\psi\rangle_{0}$ is only part of the state $|\Psi\rangle$. So $|\psi\rangle_{0}$ and $\rho_{A, m}$ with $t=t_{0}$ only catch part information of the $|\Psi\rangle$ and $\rho_{A}$. This may be the reason why $\rho_{A, m}$ can be taken as the an approximate state $\rho_{A}$ only if the probes are located in a small region of $A$ and far away from the boundary. At present we still don't 
know how to find the corrections of $|\psi\rangle_{0}$ and $\rho_{A, m}$ to make them to be better approximate states of $|\Psi\rangle$ and $\rho_{A}$.

At last, let's see the microcanonical ensemble state $\rho_{A, m}$ with $t \neq t_{0}$ in the single interval example. By using (2.37) we see that the one-point functions of quasi-primary operators $\mathcal{X}$ is non-vanishing for $t \neq b$. For example, the one-point function of $T$ and $\mathcal{A}$ is given by (2.27) and (2.30). In [14] we find a series of conditions associated with the one-point functions of quasi-primary operators for geometric states $\rho$. The first condtion is

$$
\langle\mathcal{A}\rangle_{\rho}-\langle T\rangle_{\rho}^{2} \sim O(c)
$$

if $\langle T\rangle_{\rho} \sim O(c)$. One could check the state $\rho_{A, m}$ satisfies the above condition. By using the results in appendix $\mathrm{A}$ one can further show $\rho_{A, m}$ satisfies the conditions associated with higher order quasi-primary operators $(\mathcal{X}=\mathcal{B}, \mathcal{D})$. Therefore, this suggests the microcanonical ensemble state $\rho_{A, m}$ for any $t$ may be taken as the reduced density matrix of $A$ of some pure geometric state.

In the references [52, 53] (see also [54]) the authors discuss the so-called fixed-area states in the bulk side of AdS/CFT by using the quantum-error correcting code. A noticable fact is the Rényi entropy in the fixed-area state is independent with $n$ at the leading order of $G^{-1}$ or $O(c)$. This property is similar with our microcanonical ensemble state $\rho_{A, m}$ constructed by field theory side. It is interesting to check whether $\rho_{A, m}$ has some relations to the fixed-area state.

\section{Acknowledgments}

I would like to thank Jiang Long for useful discussions. I am supported by the Fundamental Research Funds for the Central Universities under Grants NO.2020kfyXJJS041.

\section{A More examples of one-point functions of quasi-primary operators}

In section 2.2.2 we only calculate the one-point functions of $T$ and $\mathcal{A}$. Here we will study more quasi-primary operators in the vacuum conformal family. There are two quasi-primary operators with conformal dimension 6 , that is

$$
\begin{aligned}
\mathcal{B} & =(\partial T \partial T)-\frac{4}{5}\left(\partial^{2} T T\right)-\frac{1}{42} \partial^{4} T \\
\mathcal{D} & =(T(T T))-\frac{9}{10}\left(\partial^{2} T T\right)-\frac{1}{28} \partial^{4} T+\frac{93}{70 c+29} \mathcal{B} .
\end{aligned}
$$

By using the same conformal map and the transformation law of $\mathcal{B}$ and $\mathcal{D}$ we have

$$
\begin{aligned}
\langle\mathcal{B}\rangle_{\mathcal{R}_{n}} & =\frac{2 c\left(n^{2}-1\right)^{2}\left(2(35 c+61) n^{2}-93\right)}{1575 n^{6}} \frac{R^{6}}{\left(R^{2}-w^{2}\right)^{6}}, \\
\langle\mathcal{D}\rangle_{\mathcal{R}_{n}} & =\frac{c(2 c-1)(5 c+22)(7 c+68)\left(n^{2}-1\right)^{3}}{216(70 c+29) n^{6}} \frac{R^{6}}{\left(R^{2}-w^{2}\right)^{6}} .
\end{aligned}
$$


With the above results we can calculate the one-point functions

$$
\begin{gathered}
\mathcal{P}_{\mathcal{B}}(t)=\frac{2 c(70 c+122)}{1575} \frac{R^{6}}{\left(R^{2}-w^{2}\right)^{6}} \delta(t)+\frac{2 c}{1575 b^{3}} \frac{R^{6}}{\left(R^{2}-w^{2}\right)^{6}} \frac{\sqrt{b}}{\sqrt{t} I_{1}(2 \sqrt{b t})}\left[2 b^{3}(35 c+61)\right. \\
\left.\left.-b^{2}(140 c+337) t+14 b(5 c+22) t^{2} \frac{I_{3}(2 \sqrt{b t})}{I_{1}(2 \sqrt{b t})}-93 t^{3} \frac{I_{5}(2 \sqrt{b t})}{I_{1}(2 \sqrt{b t})}\right], \quad \text { (A. } 4\right)
\end{gathered}
$$

and

$$
\begin{aligned}
\mathcal{P}_{\mathcal{D}}(t)= & \frac{c(2 c-1)(5 c+22)(7 c+68)}{216(70 c+29)} \frac{R^{6}}{\left(R^{2}-w^{2}\right)^{6}} \delta(t)+\frac{c(2 c-1)(5 c+22)(7 c+68)}{216(70 c+29)} \frac{R^{6}}{\left(R^{2}-w^{2}\right)^{6}} \\
& \times \frac{\sqrt{b} I_{1}(2 \sqrt{b t})}{\sqrt{t}}\left[\left(1-\frac{3 t}{b}\right)+\frac{3 t I_{3}(2 \sqrt{b t})}{b I_{1}(2 \sqrt{b t})}-\frac{t^{2} I_{5}(2 \sqrt{b t})}{b^{2} I_{1}(2 \sqrt{b t})}\right]
\end{aligned}
$$

In the large $c$ or $b$ limit one could find an approximate expression for $\mathcal{P}_{\mathcal{D}}(t)$ and $\mathcal{P}_{\mathcal{D}}(t)$,

$$
\begin{aligned}
\mathcal{P}_{\mathcal{B}}(t) \simeq & \frac{2 c(70 c+122)}{1575} \frac{R^{6}}{\left(R^{2}-w^{2}\right)^{6}} \delta(t)+\frac{2 c}{1575} \frac{R^{6}}{\left(R^{2}-w^{2}\right)^{6}} \frac{\sqrt{b} I_{1}(2 \sqrt{b t})}{\sqrt{t}}\left[2(35 c+61)-93 \frac{t}{b}\right]\left(1-\frac{t}{b}\right)^{2}, \\
\mathcal{P}_{\mathcal{D}}(t)= & \frac{c(2 c-1)(5 c+22)(7 c+68)}{216(70 c+29)} \frac{R^{6}}{\left(R^{2}-w^{2}\right)^{6}} \delta(t)+\frac{c(2 c-1)(5 c+22)(7 c+68)}{216(70 c+29)} \frac{R^{6}}{\left(R^{2}-w^{2}\right)^{6}} \\
& \times \frac{\sqrt{b} I_{1}(2 \sqrt{b t})}{\sqrt{t}}\left(1-\frac{t}{b}\right)^{3} .
\end{aligned}
$$

$\mathcal{P}_{\mathcal{B}}(t)$ and $\mathcal{P}_{\mathcal{D}}(t)$ show similar properties as $\mathcal{P}_{T}(t)$ and $\mathcal{P}_{\mathcal{A}}(t)$. Specially, $\mathcal{P}_{\mathcal{B}}(t), \mathcal{P}_{\mathcal{D}}(t) \rightarrow 0$ when $t=b$ in the large $c$ limit.

\section{B Higher order corrections of two-point functions}

In section 2.2.5 we calculate two-point functions $\mathcal{P}_{O O}(t)$ upto order $O\left(x^{2-2 h_{\mathcal{O}}}\right)$. Here we list more higher order terms, We have

$$
\begin{aligned}
& \langle\mathcal{O}(x) \mathcal{O}(-x)\rangle_{\mathcal{R}_{n}}=(2 x)^{-2 h_{\mathcal{O}}}\left(1+\frac{h_{\mathcal{O}}\left(n^{2}-1\right)(2 x)^{2}}{3 n^{2} R^{2}}+\frac{h_{\mathcal{O}}\left(n^{2}-1\right)\left(5 h_{\mathcal{O}} n^{2}+4 n^{2}-5 h_{\mathcal{O}}-1\right)(2 x)^{4}}{90 n^{4} R^{4}}\right. \\
& +\frac{h_{\mathcal{O}}\left(n^{2}-1\right)\left(280 h_{\mathcal{O}}^{2} n^{4}-560 h_{\mathcal{O}}^{2} n^{2}+280 h_{\mathcal{O}}^{2}+672 h_{\mathcal{O}} n^{4}-840 h_{\mathcal{O}} n^{2}+168 h_{\mathcal{O}}+347 n^{4}-136 n^{2}+32\right)(2 x)^{6}}{45360 n^{6} R^{6}} \\
& \left.+o\left(x^{6}\right)\right) .
\end{aligned}
$$

With the above results we can derive

$$
\begin{aligned}
\mathcal{P}_{\mathcal{O}}(t)= & \mathcal{P}(t)(2 x)^{-2 h_{\mathcal{O}}}\left\{1+\frac{h_{\mathcal{O}}(2 x)^{2}}{3 R^{2}}\left(1-\frac{t}{b}\right)+\left[5 h_{\mathcal{O}}+4-5\left(2 h_{\mathcal{O}}+1\right) \frac{t}{b}+\left(5 h_{\mathcal{O}}+1\right) \frac{I_{3}(2 \sqrt{b t})}{I_{1}(2 \sqrt{b t})} \frac{t^{2}}{b^{2}}\right]\right. \\
& \times \frac{h_{\mathcal{O}}(2 x)^{4}}{90 R^{4}}+\left[280 h_{\mathcal{O}}^{2}+672 h_{\mathcal{O}}+347-21\left(40 h_{\mathcal{O}}^{2}+72 h_{\mathcal{O}}+23\right) \frac{t}{b}+168\left(5 h_{\mathcal{O}}^{2}+6 h_{\mathcal{O}}+1\right)\right. \\
& \left.\left.\times \frac{I_{3}(2 \sqrt{b t})}{I_{1}(2 \sqrt{b t})}\left(\frac{t}{b}\right)^{2}-8\left(35 h_{\mathcal{O}}^{2}+21 h_{\mathcal{O}}+4\right) \frac{I_{5}(2 \sqrt{b t})}{I_{1}(2 \sqrt{b t})}\left(\frac{t}{b}\right)^{3}\right] \frac{h_{\mathcal{O}}(2 x)^{6}}{45360 R^{6}}+o\left(x^{6}\right)\right\} .
\end{aligned}
$$


If we take the large $c$ limit of the above result, one would find

$$
\begin{aligned}
& \mathcal{P}_{\mathcal{O}}(t)=\mathcal{P}(t)(2 x)^{-2 h_{\mathcal{O}}}+\mathcal{P}(t)(2 x)^{-2 h_{\mathcal{O}}}\left(1-\frac{t}{b}\right)\left[\frac{h_{\mathcal{O}}(2 x)^{2}}{3 R^{2}}+\left(\left(5 h_{\mathcal{O}}+4\right)-\frac{\left(5 h_{\mathcal{O}}+1\right) t}{b}\right) \frac{h_{\mathcal{O}}(2 x)^{4}}{90 R^{4}}\right. \\
& \left.+\left(347+672 h_{\mathcal{O}}+280 h_{\mathcal{O}}^{2}-8\left(17+105 h_{\mathcal{O}}+70 h_{\mathcal{O}}^{2}\right) \frac{t}{b}+8\left(4+21 h_{\mathcal{O}}+35 h_{\mathcal{O}}^{2}\right)\left(\frac{t}{b}\right)^{2}\right) \frac{h_{\mathcal{O}}(2 x)^{6}}{45360 R^{6}}+o\left(x^{6}\right)\right] .
\end{aligned}
$$

The two-point functions are very simple upto $O\left(x^{6}\right)$ if $t=b$, that is

$$
\mathcal{P}_{\mathcal{O O}}(t=b)=\mathcal{P}(b)(2 x)^{-2 h_{\mathcal{O}}}+o\left(x^{6}\right),
$$

or

$$
\overline{\mathcal{P}}_{\mathcal{O O}}(t=b)=(2 x)^{-2 h_{\mathcal{O}}}+o\left(x^{6}\right)
$$

\section{Calculations for $d=4$}

In this section we will show the detial calculation of the density of eigenstates for dimension $d=4$. The Rényi entropy is

$$
S_{A}^{(n)}(d=4)=\frac{40 n^{4}-4\left(2 \sqrt{8 n^{2}+1}+3\right) n^{2}-\sqrt{8 n^{2}+1}-1}{128 \pi G(n-1) n^{3}} \log \left(\frac{2 R}{\epsilon}\right) .
$$

We have

$$
b:=S^{\infty}=\lim _{n \rightarrow \infty} S_{A}^{(n)}(d=4)=\frac{5}{16 \pi G} \log \frac{2 R}{\epsilon}, \quad S\left(\rho_{A}\right):=\lim _{n \rightarrow 1} S_{A}^{(n)}(d=4)=\frac{1}{2 \pi G} \log \frac{2 R}{\epsilon} .
$$

The density of eigenstates is given by

$$
\mathcal{P}(t)=\frac{1}{2 \pi i} \int_{\gamma-i \infty}^{\gamma+i \infty} d n e^{s_{n}}
$$

where

$$
s_{n}=n t+n S^{\infty}+(1-n) S_{A}^{(n)}(d=4),
$$

In the semi-classical limit $G \rightarrow 0$, we assume $t \sim O\left(G^{-1}\right)$. The condition of the saddle point approximation $\partial_{n} s_{n}=0$ gives

$$
n=\sqrt{\frac{3 b+\sqrt{15} \sqrt{\mathrm{bt}}}{10 t}} .
$$

Taking the above formula back to $s_{n}$ (C.4) we have

$$
\mathcal{P}(t)=e^{s_{n, \max }},
$$

with

$$
\begin{aligned}
s_{n, \max }= & \sqrt{\frac{1}{10} t(\sqrt{15 b t}+3 b)}+\frac{1}{2} \sqrt{\frac{5}{2}} b\left(\frac{t}{\sqrt{15 b t}+3 b}\right)^{3 / 2} \\
& \times\left(\sqrt{\frac{4(\sqrt{15 b t}+3 b)}{5 t}+1}+\frac{2(\sqrt{15 b t}+3 b)\left(2 \sqrt{\frac{4(\sqrt{15 b t}+3 b)}{5 t}+1}+3\right)}{5 t}+1\right) .
\end{aligned}
$$


In the large $t$ limit the density of eigenstates $\mathcal{P}(t) \sim e^{t^{3 / 4}}$ consistent with the result in [22]. We are interested in the case for which $\mathcal{P}\left(t_{0}\right) \simeq e^{S\left(\rho_{A}\right)}$. The non-trivial solution of $s_{n, \max }=$ $S\left(\rho_{A}\right)$ is

$$
t_{0}=\frac{3 b}{5}
$$

Taking $t_{0}$ into (C.5) we find $n=1$. Moreover, we also have $t_{0}=S\left(\rho_{A}\right)-S^{\infty}$, which is consistent with our general results in section 3.2.2.

\section{Review of short interval expansion}

In this section we briefly review the short interval expansion of the EE and Holevo information. One could refer to $[14,24,33]$ for more details. If we only consider the contributions from the vacuum conformal family, the EE of $A$ with length $\ell$ is given by

$$
\begin{aligned}
S_{A}= & \frac{c}{6} \log \frac{\ell}{\epsilon}+\ell^{2} a_{T}\langle T\rangle_{\rho}+\ell^{4} a_{T T}\langle T\rangle_{\rho}^{2}+\ell^{6} a_{T T T}\langle T\rangle_{\rho}^{3} \\
& +\ell^{8}\left(a_{\mathcal{A A}}\langle A\rangle_{\rho}^{2}+a_{T T \mathcal{A}}\langle T\rangle_{\rho}^{2}\langle\mathcal{A}\rangle_{\rho}+a_{T T T T}\langle T\rangle_{\rho}^{4}\right) \\
& +\ell^{10}\left(a_{T \mathcal{A} \mathcal{A}}\langle T\rangle_{\rho}\langle\mathcal{A}\rangle_{\rho}^{2}+a_{T T T \mathcal{A}}\langle T\rangle_{\rho}^{3}\langle\mathcal{A}\rangle_{\rho}+a_{T T T T T}\langle T\rangle_{\rho}^{5}\right)+F(\partial)+O\left(\ell^{11}\right),
\end{aligned}
$$

where the coefficients $a_{\mathcal{X}_{1} \ldots \mathcal{X}_{m}}$ are constants. We list them as follows

$$
\begin{aligned}
& a_{T}=-\frac{1}{6}, \quad a_{T T}=-\frac{1}{30 c}, \quad a_{T T T}=-\frac{4}{315 c^{2}}, \quad a_{T T \mathcal{A}}=\frac{1}{315 c^{2}}, \\
& a_{\mathcal{A A}}=-\frac{1}{126 c(5 c+22)}, \quad a_{T T T T}=-\frac{c+8}{630 c^{3}}, \quad a_{T \mathcal{A} \mathcal{A}}=-\frac{16}{693 c^{2}(5 c+22)}, \\
& a_{T T T \mathcal{A}}=\frac{32}{3465 c^{3}}, \quad a_{\text {TTTTT }}=-\frac{16(c+5)}{3465 c^{4}} .
\end{aligned}
$$

$F(\partial)$ denotes the terms with derivatives $\partial^{m}\langle\mathcal{X}\rangle_{\rho}$. For example, at order of $O\left(\ell^{3}\right)$ the term is $\frac{a_{T}}{2} \partial\langle T\rangle_{\rho}$. These terms are vanishing for translation invariant states. Though in our discussion $F(\partial)$ is non-vanishing, they are not important for our results in section 4.4.

By the definition of Holevo information (4.2) one could calculate the Holevo information of $\rho=\sum_{i} p_{i} \rho_{i}$ by short interval method. The results are given by

$$
\begin{aligned}
\chi\left(\rho_{A}\right)= & \ell^{4} a_{T T}\left(\langle T\rangle_{\rho}^{2}-\sum_{i} p_{i}\langle T\rangle_{i}^{2}\right)+\ell^{6} a_{T T T}\left(\langle T\rangle_{\rho}^{3}-\sum_{i} p_{i}\langle T\rangle_{i}^{3}\right) \\
& +\ell^{8}\left[a_{\mathcal{A A}}\left(\langle A\rangle_{\rho}^{2}-\sum_{i} p_{i}\langle A\rangle_{i}^{2}\right)+a_{T T \mathcal{A}}\left(\langle T\rangle_{\rho}^{2}\langle\mathcal{A}\rangle_{\rho}-\sum_{i} p_{i}\langle T\rangle_{i}^{2}\langle\mathcal{A}\rangle_{i}\right)\right. \\
& \left.+a_{T T T T}\left(\langle T\rangle_{\rho}^{4}-\sum_{i}\langle T\rangle_{i}^{4}\right)\right]+\ell^{10}\left[a_{T \mathcal{A} \mathcal{A}}\left(\langle T\rangle_{\rho}\langle\mathcal{A}\rangle_{\rho}^{2}-\sum_{i} p_{i}\langle T\rangle_{i}\langle\mathcal{A}\rangle_{i}^{2}\right)\right. \\
& \left.+a_{T T T \mathcal{A}}\left(\langle T\rangle_{\rho}^{3}\langle\mathcal{A}\rangle_{\rho}-\sum_{i} p_{i}\langle T\rangle_{i}^{3}\langle\mathcal{A}\rangle_{i}\right)+a_{T T T T T}\left(\langle T\rangle_{\rho}^{5}-\sum_{i} p_{i}\langle T\rangle_{i}^{5}\right)\right]+G(\partial)+O\left(\ell^{11}\right) .
\end{aligned}
$$

where $G(\partial)$ denotes the terms associated with $\partial^{m}\langle\mathcal{X}\rangle_{i}$ and $\langle\mathcal{X}\rangle_{i}:=\operatorname{tr}\left(\mathcal{X} \rho_{i}\right)$ with $\mathcal{X}=T, \mathcal{A}$.

Open Access. This article is distributed under the terms of the Creative Commons Attribution License (CC-BY 4.0), which permits any use, distribution and reproduction in any medium, provided the original author(s) and source are credited. 


\section{References}

[1] S. Ryu and T. Takayanagi, Holographic derivation of entanglement entropy from AdS/CFT, Phys. Rev. Lett. 96 (2006) 181602 [hep-th/0603001] [InSPIRE].

[2] M. Van Raamsdonk, Comments on quantum gravity and entanglement, arXiv:0907.2939 [INSPIRE].

[3] M. Van Raamsdonk, Building up spacetime with quantum entanglement, Int. J. Mod. Phys. D 19 (2010) 2429 [Gen. Rel. Grav. 42 (2010) 2323] [arXiv:1005.3035] [INSPIRE].

[4] B. Swingle, Entanglement renormalization and holography, Phys. Rev. D 86 (2012) 065007 [arXiv:0905.1317] [INSPIRE].

[5] P. Hayden, M. Headrick and A. Maloney, Holographic mutual information is monogamous, Phys. Rev. D 87 (2013) 046003 [arXiv:1107.2940] [InSPIRE].

[6] M. Botta-Cantcheff, P. Martínez and G.A. Silva, On excited states in real-time AdS/CFT, JHEP 02 (2016) 171 [arXiv: 1512.07850] [INSPIRE].

[7] M. Botta-Cantcheff, P.J. Martínez and G.A. Silva, Interacting fields in real-time AdS/CFT, JHEP 03 (2017) 148 [arXiv: 1703.02384] [INSPIRE].

[8] D. Marolf, O. Parrikar, C. Rabideau, A. Izadi Rad and M. Van Raamsdonk, From Euclidean sources to Lorentzian spacetimes in holographic conformal field theories, JHEP 06 (2018) 077 [arXiv:1709.10101] [INSPIRE].

[9] A. Belin, A. Lewkowycz and G. Sárosi, The boundary dual of the bulk symplectic form, Phys. Lett. B 789 (2019) 71 [arXiv:1806.10144] [INSPIRE].

[10] M. Botta-Cantcheff, P.J. Martínez and G.A. Silva, Holographic excited states in AdS black holes, JHEP 04 (2019) 028 [arXiv: 1901.00505] [INSPIRE].

[11] H.Z. Chen and M. Van Raamsdonk, Holographic CFT states for localized perturbations to AdS black holes, JHEP 08 (2019) 062 [arXiv: 1903.00972] [INSPIRE].

[12] A. Belin and B. Withers, From sources to initial data and back again: on bulk singularities in Euclidean AdS/CFT, JHEP 12 (2020) 185 [arXiv:2007.10344] [INSPIRE].

[13] R. Arias, M. Botta-Cantcheff, P.J. Martinez and J.F. Zarate, Modular Hamiltonian for holographic excited states, Phys. Rev. D 102 (2020) 026021 [arXiv:2002.04637] [INSPIRE].

[14] W.-Z. Guo, F.-L. Lin and J. Zhang, Nongeometric states in a holographic conformal field theory, Phys. Rev. D 99 (2019) 106001 [arXiv:1806.07595] [INSPIRE].

[15] H. Li and F. Haldane, Entanglement spectrum as a generalization of entanglement entropy: identification of topological order in non-Abelian fractional quantum Hall effect states, Phys. Rev. Lett. 101 (2008) 010504 [arXiv:0805.0332] [INSPIRE].

[16] P. Calabrese and A. Lefevre, Entanglement spectrum in one-dimensional systems, Phys. Rev. A 78 (2008) 032329 [arXiv:0806.3059].

[17] J. Cardy and E. Tonni, Entanglement hamiltonians in two-dimensional conformal field theory, J. Stat. Mech. 1612 (2016) 123103 [arXiv: 1608.01283] [INSPIRE].

[18] P. Kraus and A. Maloney, A Cardy formula for three-point coefficients or how the black hole got its spots, JHEP 05 (2017) 160 [arXiv: 1608.03284] [INSPIRE]. 
[19] A. Romero-Bermúdez, P. Sabella-Garnier and K. Schalm, A Cardy formula for off-diagonal three-point coefficients; or, how the geometry behind the horizon gets disentangled, JHEP 09 (2018) 005 [arXiv: 1804.08899] [INSPIRE].

[20] E.M. Brehm, D. Das and S. Datta, Probing thermality beyond the diagonal, Phys. Rev. D 98 (2018) 126015 [arXiv:1804.07924] [INSPIRE].

[21] X. Dong, The gravity dual of Rényi entropy, Nature Commun. 7 (2016) 12472 [arXiv: 1601.06788] [INSPIRE].

[22] L.-Y. Hung, R.C. Myers, M. Smolkin and A. Yale, Holographic calculations of Rényi entropy, JHEP 12 (2011) 047 [arXiv: 1110.1084] [InSPIRE].

[23] B. Chen and J.-J. Zhang, On short interval expansion of Rényi entropy, JHEP 11 (2013) 164 [arXiv: 1309.5453] [INSPIRE].

[24] B. Chen, J. Long and J.-J. Zhang, Holographic Rényi entropy for CFT with $W$ symmetry, JHEP 04 (2014) 041 [arXiv: 1312.5510] [INSPIRE].

[25] J. Zhang, P. Ruggiero and P. Calabrese, Subsystem trace distance in quantum field theory, Phys. Rev. Lett. 122 (2019) 141602 [arXiv:1901.10993] [INSPIRE].

[26] J. Zhang, P. Ruggiero and P. Calabrese, Subsystem trace distance in low-lying states of $(1+1)$-dimensional conformal field theories, JHEP 10 (2019) 181 [arXiv:1907.04332] [INSPIRE].

[27] D.D. Blanco, H. Casini, L.-Y. Hung and R.C. Myers, Relative entropy and holography, JHEP 08 (2013) 060 [arXiv: 1305.3182] [INSPIRE].

[28] V. Balasubramanian, J.J. Heckman and A. Maloney, Relative entropy and proximity of quantum field theories, JHEP 05 (2015) 104 [arXiv: 1410.6809] [INSPIRE].

[29] N. Lashkari, Relative entropies in conformal field theory, Phys. Rev. Lett. 113 (2014) 051602 [arXiv: 1404.3216] [INSPIRE].

[30] N. Lashkari, Modular hamiltonian for excited states in conformal field theory, Phys. Rev. Lett. 117 (2016) 041601 [arXiv: 1508.03506] [INSPIRE].

[31] G. Sárosi and T. Ugajin, Relative entropy of excited states in two dimensional conformal field theories, JHEP 07 (2016) 114 [arXiv: 1603.03057] [INSPIRE].

[32] G. Sárosi and T. Ugajin, Relative entropy of excited states in conformal field theories of arbitrary dimensions, JHEP 02 (2017) 060 [arXiv:1611.02959] [INSPIRE].

[33] W.-Z. Guo, F.-L. Lin and J. Zhang, Distinguishing black hole microstates using Holevo information, Phys. Rev. Lett. 121 (2018) 251603 [arXiv:1808.02873] [INSPIRE].

[34] X. Dong, Holographic Rényi entropy at high energy density, Phys. Rev. Lett. 122 (2019) 041602 [arXiv: 1811.04081] [INSPIRE].

[35] W.-Z. Guo, F.-L. Lin and J. Zhang, Rényi entropy at large energy density in $2 D$ CFT, JHEP 08 (2019) 010 [arXiv: 1812.11753] [INSPIRE].

[36] M. Headrick, Entanglement Rényi entropies in holographic theories, Phys. Rev. D 82 (2010) 126010 [arXiv: 1006.0047 ] [INSPIRE].

[37] J.L. Cardy, O.A. Castro-Alvaredo and B. Doyon, Form factors of branch-point twist fields in quantum integrable models and entanglement entropy, J. Statist. Phys. 130 (2008) 129 [arXiv:0706.3384] [INSPIRE]. 
[38] P. Calabrese, J. Cardy and E. Tonni, Entanglement entropy of two disjoint intervals in conformal field theory II, J. Stat. Mech. 1101 (2011) P01021 [arXiv: 1011.5482] [INSPIRE].

[39] A. Belin, A. Maloney and S. Matsuura, Holographic phases of Rényi entropies, JHEP 12 (2013) 050 [arXiv:1306.2640] [INSPIRE].

[40] L.-Y. Hung, R.C. Myers and M. Smolkin, Twist operators in higher dimensions, JHEP 10 (2014) 178 [arXiv: 1407.6429] [INSPIRE].

[41] N. Bao and H. Ooguri, Distinguishability of black hole microstates, Phys. Rev. D 96 (2017) 066017 [arXiv: 1705.07943] [INSPIRE].

[42] B. Schumacher, M. Westmoreland and W.K. Wootters, Limitation on the amount of accessible information in a quantum channel, Phys. Rev. Lett. 76 (1996) 3452.

[43] B. Czech, J.L. Karczmarek, F. Nogueira and M. Van Raamsdonk, The gravity dual of a density matrix, Class. Quant. Grav. 29 (2012) 155009 [arXiv:1204.1330] [INSPIRE].

[44] A.C. Wall, Maximin surfaces, and the strong subadditivity of the covariant holographic entanglement entropy, Class. Quant. Grav. 31 (2014) 225007 [arXiv:1211.3494] [INSPIRE].

[45] M. Headrick, V.E. Hubeny, A. Lawrence and M. Rangamani, Causality \& holographic entanglement entropy, JHEP 12 (2014) 162 [arXiv:1408.6300] [INSPIRE].

[46] X. Dong, D. Harlow and A.C. Wall, Reconstruction of bulk operators within the entanglement wedge in gauge-gravity duality, Phys. Rev. Lett. 117 (2016) 021601 [arXiv:1601.05416] [INSPIRE].

[47] T. Takayanagi and K. Umemoto, Entanglement of purification through holographic duality, Nature Phys. 14 (2018) 573 [arXiv:1708. 09393] [INSPIRE].

[48] P. Nguyen, T. Devakul, M.G. Halbasch, M.P. Zaletel and B. Swingle, Entanglement of purification: from spin chains to holography, JHEP 01 (2018) 098 [arXiv:1709.07424] [INSPIRE].

[49] W.-Z. Guo, Correlations in geometric states, JHEP 08 (2020) 125 [arXiv:2003.03933] [INSPIRE].

[50] N. Bao, G. Penington, J. Sorce and A.C. Wall, Holographic tensor networks in full $A d S / C F T$, arXiv:1902.10157 [INSPIRE].

[51] N. Bao, G. Penington, J. Sorce and A.C. Wall, Beyond toy models: distilling tensor networks in full AdS/CFT, JHEP 11 (2019) 069 [arXiv:1812.01171] [INSPIRE].

[52] X. Dong, D. Harlow and D. Marolf, Flat entanglement spectra in fixed-area states of quantum gravity, JHEP 10 (2019) 240 [arXiv:1811.05382] [INSPIRE].

[53] C. Akers and P. Rath, Holographic Rényi entropy from quantum error correction, JHEP 05 (2019) 052 [arXiv : 1811.05171] [INSPIRE].

[54] X. Dong and D. Marolf, One-loop universality of holographic codes, JHEP 03 (2020) 191 [arXiv: 1910.06329] [INSPIRE]. 